\title{
Coastal foraminifera from the Iranian coast of Makran, Oman Sea (Chabahar Bay to Gawater Bay) as an indicator of tsunamis
}

\author{
Seyed Hamid Vaziri ${ }^{1 *}$, Eduard G. Reinhart ${ }^{2}$, Jessica E. Pilarczyk ${ }^{3}$ \\ ${ }^{1}$ Department of Geology, North Tehran Branch, Islamic Azad University, P.O. Box 19585-851,Tehran, Iran \\ ${ }^{2}$ School of Geography \& Earth Sciences, McMaster University, 1280 Main Street W., Hamilton, ON, L8S 4K1, Canada \\ ${ }^{3}$ Division of Marine Science, University of Southern Mississippi, Stennis Space Center, 1020 Balch Blvd., MS 3952, USA \\ *Corresponding author, e-mail: h_vaziri@iau-tnb.ac.ir
}

(received: 31/03/2018; accepted: 29/09/2018)

\begin{abstract}
Extreme coastal inundation associated with the 2004 Indian Ocean and 1945 Makran tsunamigenic-earthquakes highlight the risk of tsunamis to coastlines of the northern Oman Sea. Foraminifera have been used as indicators of paleotsunamis in the past where allochthonous tests, found in low-energy environments such as in coastal lagoons, ponds, and marshes, indicate marine overwash. In this study, we constrain the modern distributions of foraminifera from coastal Iran so that they may be used to identify and interpret (e.g. assess provenance) paleotsunami deposits in the geologic record. We collected surface sediment samples from sixteen sites within the study area from Chabahar to Gawater Bays on the Makran coast of Iran, selecting locations impacted by the 1945 Makran tsunami. Foraminifera obtained from these locations are dominated by supratidal, intertidal, and subtidal species, with minor abundances of planktic taxa. Samples collected from study locations are characterized by abundances of iron-stained and heavily corroded (e.g. edge rounded and pitted) individuals. Cluster analysis was used to determine three foraminiferal assemblages within the Makran coastal zone: subtidal, intertidal and supratidal. Characterizing modern distributions of foraminifera along the Makran coast of Iran will aid in identifying the provenance of older overwash deposits previously identified in this region.
\end{abstract}

Keywords: Foraminifera, Coastal Sediments, Paleotsunami, Makran, Oman Sea.

\section{Introduction}

Paleotsunami deposits preserved in the geological record provide a means of extending the known record of events from several hundreds of years to several thousands of years, capturing both the frequent and infrequent, but higher magnitude, events. The majority of studies on paleotsunami deposits have been conducted in temperate regions, with few studies in tropical regions, and even fewer in arid environments. Although the 2004 Indian Ocean tsunami and the 1945 Makran tsunami demonstrate the tsunami risk to the Makran area of Iran and adjacent coastlines, this area has been the subject of few published (Mokhtari et al., 2008; Shah-Hosseini et al., 2011; Hamzeh et al., 2013;Okal et al., 2015) paleotsunami studies. Donato et al., (2008, 2009) and Pilarczyk et al., (2011, 2012) investigated shell layers, grain size distributions, and foraminifera, respectively, from the 1945 Makran tsunami in Sur Lagoon (coast of Oman). Numerical studies have also been done to assess the tsunami risk of the Makran and adjacent coastlines (Mokhtari et al., 2008; Heidarzadeh et al., 2008a, 2009; Okal \& Synolakis, 2008; Okal et al., 2015; Jaiswal et al., 2009; Shah-Hosseini et al., 2011; Payande et al., 2015).

Foraminifera are useful indicators of tsunami and storm overwash because they identify allochthonous sediments in marginal marine environments such as coastal lagoons, ponds, and marshes. Studies that employ foraminifera to identify marine overwash are abundant in temperate regions (e.g. Hippensteel et al., 2000; Scott et al., 2003; Hawkes \& Horton, 2012; Pilarczyk et al., 2012), with few studies in tropical regions (Sugawara et al., 2009; Sieh et al., 2015; Goff et al., 2011), and even fewer in arid environments (e.g. Dominey-Howes et al., 2006; Pilarczyk \& Reinhardt, 2012). Tsunami deposits in arid environments are generally poorly preserved due to high rates of deflation. The accelerated postdepositional change in arid environments results in difficulty in identifying and interpreting overwash deposits. Foraminifera help in this regard because their tests preserve well in the geologic record and their presence indicates a marine origin (Pilarczyk et al., 2014).

Studies that document the modern spatial distribution of foraminifera can be used as a basis for comparison with overwash sediments to assess sediment provenance (Kosciuch et al., 2018; Phat et al., 2018). Modern distributions of various subenvironments found in western India, Oman, Iran, and the Persian Gulf have been extensively 
docuemented (e.g. Murray, 1965, 1966a, b, c; Reddy \& Rao, 1984; EL-Nakhal, 1990; Nigam \& Khare, 1995; Cherif et al., 1997; Lezine et al., 2002; Bahalla et al., 2007; Moghaddasi et al., 2008; Ghosh et al., 2009; Pilarczyk et al., 2011).

Historical records mention several tsunamis that have impacted coastlines around the northern Arabian Sea (e.g. events at 325 BC, 1483, 1765, 1851, etc.; Ambraseys \& Melville, 1982; Heidarzadeh et al., 2008a, 2008b; Heidarzadeh et al., 2009), including at least six events that had a magnitude greater than 8.0. On 28 November 1945, a Mw 8.1 subduction zone earthquake occurred approximately $300 \mathrm{~km}$ west of Karachi, Pakistan, resulting in a tsunami with wave heights ranging from 2 to 13 m (Ambresys \& Melville, 1982; Byrne et al., 1992; Pararas-Carayannis, 2006). Tsunami run-up heights along the Iranian coast from this event were variable, ranging from $0.5 \mathrm{~m}$ in Gordim Bay near the town of Konarak to $10 \mathrm{~m}$ in Gawater Bay near the border with Pakistan (Hamzeh et al., 2013). Death tolls associated with the 1945 tsunami were low along the Iranian coast due to the low population density that was limited to small fishing villages (e.g. Chabahar, Lipar, Beris, and Pasabandar).

In this study, we document the surface distribution of foraminifera in coastal sediments along the Iranian coast between Chabahar and Gawater Bays and assess whether they can be used as indicators of tsunami overwash in this region. The modern distribution of foraminifera along the
Chabahar Bay coastline is mapped in order to determine which species and taphonomic characters (i.e., surface condition of individual foraminifera) will be most useful in detecting and interpreting overwash deposition.

\section{Regional setting}

The northwestern Makran coast is vulnerable to earthquakes and tsunamis as a result of its proximity to the Makran Subduction Zone. The coastline stretches a distances of approximately $1000 \mathrm{~km}$ between Iran and Pakistan, from the Strait of Hormoz in the west to Karachi, Pakistan in the east. This region is part of the accretionary wedge of the Makran Subduction Zone, formed by the subduction of the Arabian Plate under the Eurasian Plate (Byrne et al., 1992; Regard et al., 2005). The Makran coast is marked by a series of prominent headlands separated by bays. Further inland, uplifted Plio-Pleistocene marine terraces and sandstones are undergoing intensive erosion under arid conditions (Reyss et al., 1998). The coastline has been prograding since the mid-Holocene, owing to both the slight tectonic uplift and marine and alluvial sedimentation (Reyss et al., 1998).

The Iranian part of the Makran coast is a subtropical arid region, extending for approximately $640 \mathrm{~km}$ from Bandar Abbas in the Strait of Hormuz to Gawater on the Iranian-Pakistani border. However, this study focuses on the area between Chabahar and Gawater Bays (Fig. 1).

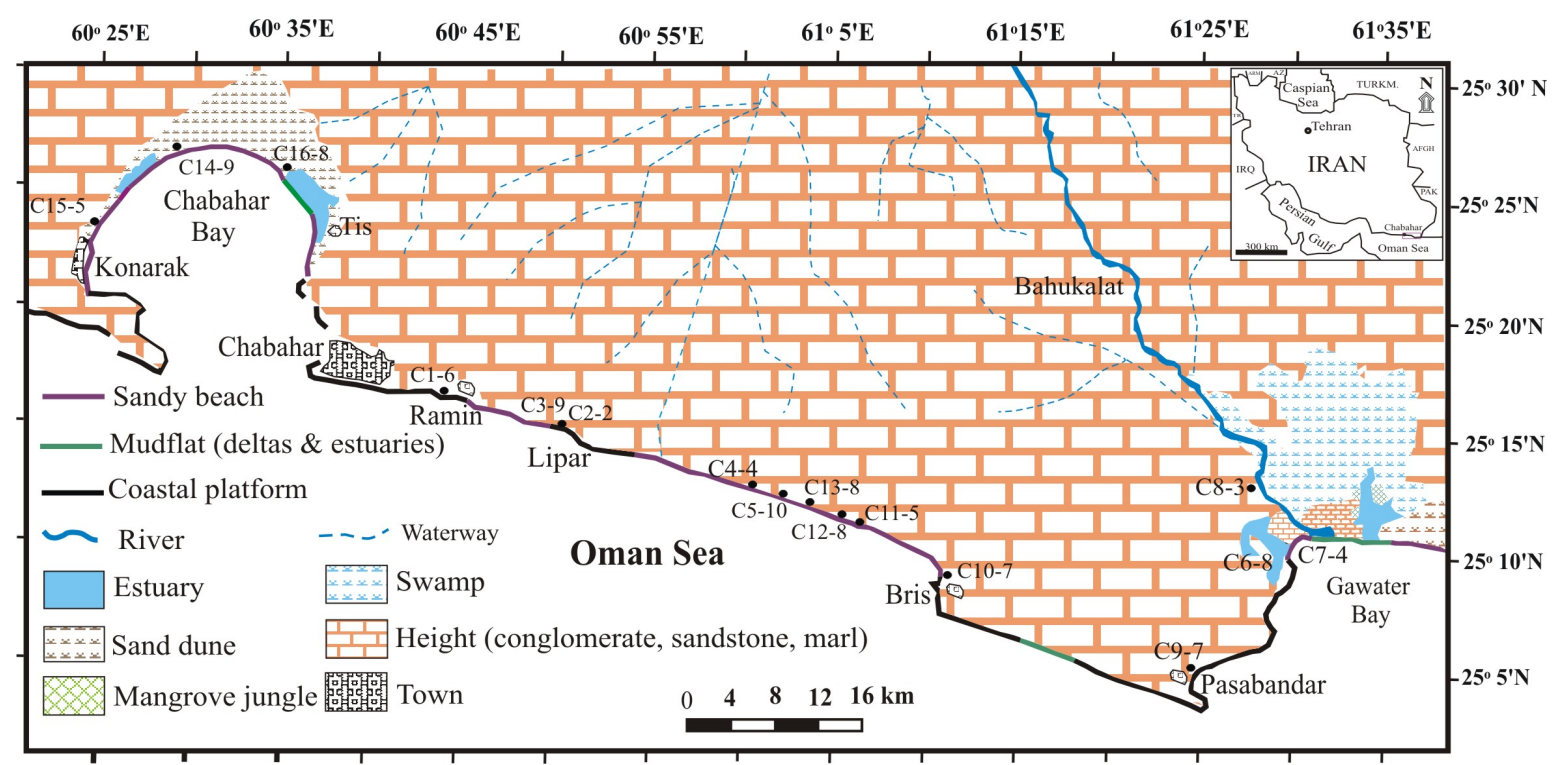

Figure 1. Geomorphology map (modified from Hamzeh et al., 2014) and locations of the study sites from Chabahar to Gawater Bay, on the Iranian Makran coast, Oman Sea. 
The Iranian coast along the Oman Sea (Sistan and Baluchestan Province) can be divided into three main geomorphologic features: coastal platforms (terraces), sand beaches, and mud flats (deltas and estuaries) (Hamzeh et al., 2014; Fig. 1). Coastal platforms are the most frequent landform in the area and are formed by the aforementioned tectonic uplift of coastal Makran and the effect of waves upon the shoreline. Onshore sandy dunes are common features in the study area. Within the bays, paleobeaches are seen as sand bands parallel to the modern shoreline. Deltas and estuaries are formed by sedimentation of seasonal streams, with mangroves formed in some estuaries, such as at Bahukalat, and in parts of Chabahar Bay (Fig. 1). In general, the geomorphology of coastal Makran is closely controlled by the coast's structural pattern, weather conditions, and geological facies (Hamzeh et al., 2013).

\section{Materials and Methods}

Sixteen sites (see Tables 1-3) along the Iranian coast of the Oman Sea were investigated for foraminiferal content. The sites are located between Chabahar Bay and the middle part of Gawater Bay at the Iran-Pakistan border (Fig. 1) and include coastal platforms, sandy beaches, and mudflats deposited in the tidal-channel levee, intertidal, and subtidal environments (Tables 1-3). From the study sites, sixteen cores (core depths $32-143 \mathrm{~cm}$ ) were collected from sandy beaches and mudflats (Figs. 1-5). In total, 109 sediment samples were collected from these cores and 16 surface samples (collected from the upper $2 \mathrm{~cm}$ of sediment) were investigated for granulometry (grain-size) and their foraminifera distributions (Tables 1-3).

Samples for foraminiferal analysis were sieved at $63 \mu \mathrm{m}$, dry split to obtain counts of approximately 300 specimens, and examined under a microscope. Specimens were picked by hand and placed on slides for identification and imaging. Taxonomy followed Debenay (2012), Loeblich \& Tapan (1988), Ellis and Messina's Foraminifera Catalogue (2010), and other previous studies (e.g. Moghaddasi et al., 2008; Debenay, 2001; Murray, 1965, 1966a, 1966b; Nigam \& Khare, 1995; Akimoto et al., 2002; Hottinger et al., 1993; Cimerman \& Langer, 1991; Bhalla et al., 2007). The taphonomic condition of each individual foraminifer was assessed and categorized according to the observation of iron-staining and degree of corrosion in three levels minor, moderate and maximum (Fig. 6). A scanning-electron microscope (SEM) located at the Canadian Centre for Electron Microscopy at McMaster University was used to take photographs of foraminiferal specimens.

Partitioning Around Medoid (PAM) cluster analysis (Kaufman \& Rousseeuw, 1990) was then used to identify biofacies within the modern environment at Chabahar and Gawater Bays. The methods of Pilarczyk et al. (2016) and Kosciuch et al. (2018) were followed, where foraminiferal counts (iron stained versus unstained foraminifera) were first converted to relative abundances, and then standardized using $\mathrm{z}$-scores. PAM cluster analysis produces silhouette widths that indicate how appropriately a given sample is clustered. A silhouette width of -1 indicates an incorrect classification, whereas a silhouette width of 1 indicates perfect assignment to the appropriate cluster. The number of biofacies at our study sites was determined by selecting the cluster scenario that produced the highest average silhouette width.

\section{Results}

Within the sampling area, three major sedimentological sub-environments exist: sandy beaches, mudflats and tidal channels. Foraminifera collected from these subenvironments were characterized by high abundances of iron staining and/or corrosion. Comprehensive data on the study sites including location, sediment, grains/size, foraminifera, corrosion etc. are showing in tables 1-3.

\section{Distribution of foraminifera}

Sixteen surface samples were selected for foraminiferal analysis. All samples contained abundant foraminifera (85-100\%) and a significant percentage of the specimens had iron stained tests (Figs. 7-10; Tables 1-3). The foraminifera identified in the samples belong to 38 genera and 41 species (Fig. 11). The most abundant species include Ammonia convexa (15-68\%), Elphidium craticulatum (6-26\%), Assilina ammonoides (17$31 \%$ ) and miliolids (3-19\%). Apart from foraminifera, other invertebrates, including ostracods, gastropods, bivalves, and sponges were also recovered from the samples.

\section{Sandy beach subenvironment}

Sandy beaches are composed of poorly to well sorted, fine to very coarse sand and are interpreted as intertidal in nature (Samples C1-6, C9-7, C13- 
8, and $\mathrm{C} 16-8)$. The most abundant foraminiferal species recovered from these samples were Ammonia convexa, Assilina ammonoides, Elphidium craticulatum and various miliolids. Other recorded invertebrates included ostracods, gastropods, and sponges. Overall, $47-60 \%$ of foraminiferal tests show iron staining, with maximum, moderate, and minor levels of corrosion seen in a range of $0-33 \%, 20-50 \%$, and $17-63 \%$ of specimens, respectively.

Table 1. Comprehensive data on the Ramin to Kochak Estuaries sites (samples C1-6 to C5-10) of the Iranian Makran coast, Oman Sea, including location, sediment, grains/size, foraminifera, and corrosion levels.

\begin{tabular}{|c|c|c|c|c|c|}
\hline Sample no. & C1-6 & $\mathrm{C} 2-2$ & C3-9 & C4-4 & C5-10 \\
\hline Location & Ramin Estuary & Lipar Estuary & Lipar Estuary & Gareendar Estuary & Kochak Estuary \\
\hline Coordinates & $\begin{array}{l}\mathrm{N} 25^{\circ} .16^{\circ} .04 .7^{\prime \prime} \\
\mathrm{E} 60^{\circ} .45^{\prime} .06 .5^{\prime \prime}\end{array}$ & $\begin{array}{l}\mathrm{N} 25^{\circ} .15^{\prime} .10 \cdot 3^{\prime \prime} \\
\mathrm{E} 60^{\circ} .49^{\prime} .51 .0^{\prime \prime}\end{array}$ & $\begin{array}{l}\text { N } 25^{\circ} .15^{\prime} .07 .1^{\prime \prime} \\
\text { E } 60^{\circ} .49^{\prime} .45 .4^{\prime \prime}\end{array}$ & $\begin{array}{l}\mathrm{N} 25^{\circ} .13^{\circ} .05 .1^{\prime \prime} \\
\mathrm{E} 60^{\circ} .59^{\prime} .04 .1^{\prime \prime}\end{array}$ & $\begin{array}{l}\mathrm{N} 25^{\circ} .12^{\prime} .37 .9^{\prime \prime} \\
\text { E } 61^{\circ} .01^{\prime} .0 .2^{\prime \prime}\end{array}$ \\
\hline Environment & Intertidal & $\begin{array}{c}\text { Supratidal, Tidal } \\
\text { channel }\end{array}$ & $\begin{array}{l}\text { Supratidal, Tidal } \\
\text { channel levee }\end{array}$ & $\begin{array}{c}\text { Supratidal, Tidal } \\
\text { channel levee }\end{array}$ & $\begin{array}{l}\text { Supratidal, Tidal } \\
\text { channel levee }\end{array}$ \\
\hline Sediments & Costal sand & Sand & Sand & Sand & Sand \\
\hline Grains/Size & $\begin{array}{l}\text { Medium, coarse-very } \\
\text { coarse sand }\end{array}$ & Fine silt and sand & Fine to coarse sand & $\begin{array}{l}\text { Medium to coarse } \\
\text { sand }\end{array}$ & Medium sand \\
\hline Sorting & Poorly sorted & Poorly sorted & $\begin{array}{c}\text { Relatively poorly } \\
\text { sorted }\end{array}$ & Fairly well sorted & Well sorted \\
\hline Foraminifera & $\begin{array}{c}(98.4 \%) \\
\text { Ammonia convexa } \\
\text { Ammonia aoteana } \\
\text { Anomalinulla glabrata } \\
\text { Assilina ammonoides } \\
\text { Bolivina striatula } \\
\text { Elphidium macellum } \\
\text { Elphidium crispum } \\
\text { Globigerinoides } \mathrm{sp} . \\
\text { Gyroidina } \mathrm{sp} . \\
\text { Lenticulina imbosa } \\
\text { Lenticulina tasmanica } \\
\text { Monspeliensina } \mathrm{sp} . \\
\text { Pararotalia } \mathrm{sp} .\end{array}$ & $\begin{array}{c}(100 \%) \\
\text { Ammonia convexa } \\
\text { Ammonia aoteana } \\
\text { Amphistegina lessonii } \\
\text { Assilina ammonoides } \\
\text { Bolivina striatula } \\
\text { Elphidium craticulatum } \\
\text { Elphidium crispum } \\
\text { Globigcrinita } \mathrm{sp} . \\
\text { Lagena } \mathrm{sp} . \\
\text { Lenticulina imbosa } \\
\text { Nodosaria } \mathrm{sp} . \\
\text { Purarotalia } \mathrm{sp} . \\
\text { Perneroplis pertusus } \\
\text { Rosalina orientalis } \\
\text { Siphogenerina } \mathrm{sp} ., \\
\text { Miliolids }\end{array}$ & $\begin{array}{c}(89.5 \%) \\
\text { Ammonia convexa } \\
\text { Ammonia aoteana } \\
\text { Amphistegina } \\
\text { lessonii } \\
\text { Assilina } \\
\text { ammonoides } \\
\text { Elphidium } \\
\text { craticulatum } \\
\text { Elphidium crispum } \\
\text { Globorotalia sp. } \\
\text { Lenticulina sp. } \\
\text { Lenticulina cultrate } \\
\text { Lenticulina imbosa } \\
\text { Neocassidulina } \\
\text { abbreviate } \\
\text { Siphogenerina } \\
\text { raphana } \\
\text { Uvigerina schencki } \\
\text { Virgulinella sp. } \\
\text { Miliolids }\end{array}$ & $\begin{array}{c}\text { (99.3\%) } \\
\text { Ammonia convexa } \\
\text { Ammonia aoteana } \\
\text { Ammonia tepida } \\
\text { Ammonia inflata } \\
\text { Assilina ammonoides } \\
\text { Bolivina striatula } \\
\text { Challengerella } \text { sp., } \\
\text { Cibicides sp. } \\
\text { Elphidium } \\
\text { craticulatum } \\
\text { Globigerinoides ruber } \\
\text { Lenticulina cultrata, } \\
\text { L. imbosa } \\
\text { Nodosaria } \text { sp. } \\
\text { Laevidentalia } \\
\text { subsoluta } \\
\text { Pararotalia } \mathrm{sp} . \\
\text { Siphotextularia } \\
\text { heterastoma } \\
\text { Textularia } \mathrm{sp} ., \\
\text { Miliolids } \\
\end{array}$ & $\begin{array}{c}(100 \%) \\
\text { Ammonia convexa } \\
\text { Ammonia aoteana } \\
\text { Ammonia tepida } \\
\text { Assilina } \\
\text { ammonoides } \\
\text { Asterorotalia } \\
\text { gaimardi } \\
\text { Bolivina striatula } \\
\text { Bolivina spathulata } \\
\text { Elphidium } \\
\text { craticulatum } \\
\text { Elphidium crispum } \\
\text { Globigerinoides sp. } \\
\text { Laevidentalia } \mathrm{sp} . \\
\text { Lenticulina cultrate } \\
\text { Lenticulina imbosa } \\
\text { Pararotalia } \mathrm{sp} . \\
\text { Textularia } \mathrm{sp} ., \\
\text { Miliolids }\end{array}$ \\
\hline Other fauna & Ostracoda $(1.6 \%)$ & N/A & $\begin{array}{c}\text { Gastrapoda }(7.9 \%) \\
\text { Sponge }(2.6 \%)\end{array}$ & Gastrapoda $(0.7 \%)$ & N/A \\
\hline $\begin{array}{l}\text { The most } \\
\text { abundant } \\
\text { fauna }\end{array}$ & $\begin{array}{c}\text { Ammonia convexa } \\
(36 \%) \\
\text { Elphidium craticulatum } \\
(15.8 \%)\end{array}$ & $\begin{array}{c}\text { Ammonia convexa } \\
(19.2 \%) \\
\text { Elphidium craticulatum } \\
(5.8 \%)\end{array}$ & $\begin{array}{c}\text { Ammonia convexa } \\
(34.7 \%) \\
\text { Lenticulina sp. } \\
(11.2 \%) \text { Miliolids } \\
(11.2 \%)\end{array}$ & $\begin{array}{c}\text { Ammonia convexa } \\
(68 \%) \\
\text { Elphidium } \\
\text { craticulutum }(10.4 \%)\end{array}$ & $\begin{array}{c}\text { Ammonia convexa } \\
(51 \%) \\
\text { Ammonia aoteana } \\
(12.4 \%) \\
\text { Elphidium } \\
\text { craticulatum } \\
(12.2 \%) \\
\end{array}$ \\
\hline $\begin{array}{l}\text { Total number } \\
\text { of specimens }\end{array}$ & 186 & 216 & 213 & 480 & 680 \\
\hline $\begin{array}{l}\text { Forams with } \\
\text { iron stain }\end{array}$ & $55 \%$ & $75.8 \%$ & $60 \%$ & $69.9 \%$ & $75.4 \%$ \\
\hline $\begin{array}{c}\text { Max } \\
\text { Corrosion }\end{array}$ & $0 \%$ & $16 \%$ & $0 \%$ & $0 \%$ & $46 \%$ \\
\hline $\begin{array}{c}\text { Mod } \\
\text { Corrosion }\end{array}$ & $37 \%$ & $26 \%$ & $47 \%$ & $20 \%$ & $19 \%$ \\
\hline $\begin{array}{c}\text { Minor } \\
\text { Corrosion }\end{array}$ & $63 \%$ & $58 \%$ & $53 \%$ & $80 \%$ & $35 \%$ \\
\hline $\begin{array}{c}\text { Large } \\
\text { fragments }\end{array}$ & $52.8 \%$ & $50.4 \%$ & $35.7 \%$ & $49.3 \%$ & $61.8 \%$ \\
\hline $\begin{array}{c}\text { Small } \\
\text { fragments }\end{array}$ & $47.2 \%$ & $41.6 \%$ & $64.3 \%$ & $50.7 \%$ & $38.2 \%$ \\
\hline
\end{tabular}


Table 2. Comprehensive data on the Gawater Estuary, Pasabandar, and Beris sites (samples C6-8 to C10-7) of the Iranian Makran coast, Oman Sea, including location, sediment, grains/size, foraminifera, and corrosion levels.

\begin{tabular}{|c|c|c|c|c|c|}
\hline Sample no. & C6-8 & C7-4 & C8-3 & C9-7 & C10-7 \\
\hline Location & Gawater Estuary & Gawater Estuary & Gawater Estuary & Pasabandar & Beris Estuary \\
\hline Coordinates & $\begin{array}{l}\mathrm{N} 25^{\circ} .10^{\prime} .05 .7^{\prime \prime} \\
\mathrm{E} 61^{\circ} .29^{\prime} .47 .0^{\prime \prime}\end{array}$ & $\begin{array}{l}\text { N } 25^{\circ} .10^{\prime} .04 .1^{\prime \prime} \\
\text { E } 61^{\circ} .29^{\prime} .39 .3^{\prime \prime}\end{array}$ & $\begin{array}{l}\text { N } 25^{\circ} .12^{\prime} .33 .8^{\prime \prime} \\
\text { E } 61^{\circ} .28^{\prime} .37 .8^{\prime \prime}\end{array}$ & $\begin{array}{l}\text { N } 25^{\circ} .04^{\prime} .51 .9^{\prime \prime} \\
\text { E } 61^{\circ} .24^{\prime} .45 .3^{\prime \prime}\end{array}$ & $\begin{array}{l}\text { N } 25^{\circ} .09^{\prime} .17 .7^{\prime \prime} \\
\text { E } 61^{\circ} .10^{\prime} .47 .6^{\prime \prime}\end{array}$ \\
\hline Environment & $\begin{array}{c}\text { Subtidal, Tidal channel } \\
\text { levee }\end{array}$ & $\begin{array}{c}\text { Subtidal, Tidal channel } \\
\text { levee }\end{array}$ & $\begin{array}{c}\text { Subtidal, Tidal channel } \\
\text { levee }\end{array}$ & Intertidal & Supratidal \\
\hline Sediments & Mud (clayey) & Mud (clayey) & Mud (clayey) & Costal sand & sand \\
\hline Grains/Size & Fine silt and clay & Fine silt and clay & Mud, very fine grains & Fine to medium sand & Fine to coarse sand \\
\hline Sorting & Fairly well sorted & Well sorted & Well sorted & Relatively poorly sorted & Fairly well sorted \\
\hline Foraminifera & $\begin{array}{c}\text { (85\%) } \\
\text { Ammonia convexa } \\
\text { Ammonia aoteana } \\
\text { Anomalimulla glahrata } \\
\text { Assilina ammonoides } \\
\text { Asterorotalia gaimardi } \\
\text { Cancris auriculus } \\
\text { Cancris sagrum } \\
\text { Elphidium fichtelianum } \\
\text { Elphidium craticulatum } \\
\text { Elphidium crispum } \\
\text { Elphidium albanii } \\
\text { Eponides repandus } \\
\text { Globigerinoides sp. } \\
\text { Heterolepa } \\
\text { subhaidingeri } \\
\text { Laevidentalia } \mathrm{sp} . \\
\text { Peneroplis planatus } \\
\text { Pararotalia } \mathrm{sp} . \\
\text { Miliolids }\end{array}$ & $\begin{array}{c}\text { (99.5\%) } \\
\text { Ammonia convexa } \\
\text { Ammonia parkinsoniana } \\
\text { Assilina ammonoides } \\
\text { Asterorotalia gaimardi } \\
\text { Cancris auriculus } \\
\text { Cassidelina } \text { sp. } \\
\text { Elphidium craticulatum } \\
\text { Elphidium crispum } \\
\text { Elphidium excavatum } \\
\text { Globigerinoides sp. } \\
\text { Heterolepa subhaidingeri } \\
\text { Lenticulina imbosa } \\
\text { Pararotalia sp. } \\
\text { Pararotalia nipponica } \\
\text { Textularia sp. } \\
\text { Miliolids }\end{array}$ & $\begin{array}{c}(84.6 \%) \\
\text { Assilina ammonoides } \\
\text { Asterorotalia gaimardi } \\
\text { Bolivina spathulata } \\
\text { Pararotalia sp. } \\
\text { Pararotalia nipponica } \\
\text { Peneroplis planatus } \\
\text { Miliolids }\end{array}$ & $\begin{array}{c}\text { (97.7\%) } \\
\text { Ammonia convexa } \\
\text { Ammonia aoteana } \\
\text { Amphicoryna scalaris } \\
\text { Amphicoryna scalaris } \\
\text { Assilina ammonoides } \\
\text { Asterorotalia gaimardi } \\
\text { Bolivina spathulata } \\
\text { Discorbinella sp. } \\
\text { Elphidium craticulatum } \\
\text { E. crispum, E excavatum } \\
\text { Eponides repandus } \\
\text { Globigerinoides ruber } \\
\text { Globigerinella sp. } \\
\text { Lohatula lohatula } \\
\text { Lenticulina imbosa, L. } \\
\text { australis } \\
\text { Nonion subturgidum } \\
\text { Pararotalia sp., Rosalina } \\
\text { sp. } \\
\text { Siphogenerina sp. } \\
\text { Stomatorbina concentric } \\
\text { Textularia sp., Miliolids }\end{array}$ & $\begin{array}{c}\text { (97.2\%) } \\
\text { Ammonia convexa } \\
\text { Ammonia anteana } \\
\text { Assilina ammonoides } \\
\text { Asterorotalia gaimardi } \\
\text { Bolivina sp. } \\
\text { Bolivina spathulata } \\
\text { Cibicides } \\
\text { pseudolobatulus } \\
\text { Discorbinella rodiensis } \\
\text { Elphidium craticulatum } \\
\text { Elphidium maorium } \\
\text { Globoturborotalita? sp. } \\
\text { Lenticulina } \text { sp. } \\
\text { Pararotalia } \mathrm{sp} . \\
\text { Pararotalia nipponica } \\
\text { Textularia sp. } \\
\text { Miliolids }\end{array}$ \\
\hline Other fauna & $\begin{array}{c}\text { Ostracoda }(10.8 \%) \\
\text { Sponge }(2.7 \%) \\
\text { Gastrapoda }(1.5 \%) \\
\end{array}$ & Gastrapoda $(0.5 \%)$ & Ostracoda (15.4\%) & $\begin{array}{c}\text { Gastrapoda }(0.6 \%) \\
\text { Sponge }(1.7 \%)\end{array}$ & $\begin{array}{c}\text { Ostracoda }(0.8 \%), \\
\text { Sponge }(1.2 \%) \\
\text { Gastrapoda }(0.8 \%)\end{array}$ \\
\hline $\begin{array}{c}\text { The most } \\
\text { abundant } \\
\text { fauna }\end{array}$ & $\begin{array}{c}\text { Ammonia convexa } \\
(28.6 \%) \\
\text { Elphidium craticulatum } \\
(14.6 \%) \\
\text { Miliolids }(2.7 \%)\end{array}$ & $\begin{array}{c}\text { Ammonia convexa } \\
(42.5 \%) \text { Asterorotalia } \\
\text { galimardi }(13 \%) \\
\text { Elphidium craticulatum } \\
(12.5 \%) \\
\end{array}$ & $\begin{array}{c}\text { Pararotalia nipponica } \\
(36.4 \%) \\
\text { Asterorotalia galimardi } \\
(18.2 \%)\end{array}$ & $\begin{array}{c}\text { Ammonia convexa } \\
(14.45 \%) \\
\text { Assilina ammonoides } \\
(20.8 \%) \\
\text { Miliolids }(19 \%) \\
\end{array}$ & $\begin{array}{c}\text { Ammonia convexa } \\
(19 \%) \\
\text { Assilina ammonoides } \\
(18.2 \%) \\
\text { Miliolids }(18.6 \%)\end{array}$ \\
\hline $\begin{array}{c}\begin{array}{c}\text { Total number } \\
\text { of specimens }\end{array} \\
\end{array}$ & 204 & 236 & 15 & 254 & 394 \\
\hline $\begin{array}{c}\text { Forams with } \\
\text { iron stain }\end{array}$ & $28 \%$ & $14 \%$ & $36.4 \%$ & $46.8 \%$ & $63.5 \%$ \\
\hline $\begin{array}{c}\text { Max } \\
\text { Corrosion }\end{array}$ & $0 \%$ & $0 \%$ & $0 \%$ & $33 \%$ & $53 \%$ \\
\hline $\begin{array}{c}\text { Mod } \\
\text { Corrosion }\end{array}$ & $50 \%$ & $0 \%$ & $0 \%$ & $50 \%$ & $21 \%$ \\
\hline $\begin{array}{c}\text { Minor } \\
\text { Corrosion }\end{array}$ & $50 \%$ & $100 \%$ & $100 \%$ & $17 \%$ & $26 \%$ \\
\hline $\begin{array}{c}\text { Large } \\
\text { fragments }\end{array}$ & $42.1 \%$ & $36.8 \%$ & $100 \%$ & $49.47 \%$ & $62.36 \%$ \\
\hline $\begin{array}{c}\text { Small } \\
\text { fragments }\end{array}$ & $57.9 \%$ & $63.2 \%$ & $0 \%$ & $50.53 \%$ & $36.64 \%$ \\
\hline
\end{tabular}

Among the shell fragments (foraminifera and other fauna), large fragments account for $39-61 \%$ of the material, and small fragments making up 38$61 \%$ of specimens (Tables $1-3$ ).

\section{Mudflat subenvironment}

Mudflats, which include delta and estuarine sites, yielded sediment samples composed of poorly to well sorted, fine silt and clay. These sites are subtidal and tend to be related to tidal channel levee environments (Samples C6-8, C7-4, C8-3, C14-9, and $\mathrm{C} 15-5)$. The most abundant foraminiferal species in these samples are Ammonia convexa, Assilina ammonoides Asterorotalia galimardi, Elphidium craticulatum, Pararotalia nipponica, Spiroplectinella sp., and various miliolids. Besides foraminifera, ostracods, gastropods, bivalves, and sponges are also seen in these samples. Overall, 14$36 \%$ of foraminifera in mudflat samples have iron stained tests, with maximum, moderate, and minor 
levels of corrosion recorded in a range of $0-22 \%$, $0-56 \%$, and $22-100 \%$ of specimens, respectively. Among the shell fragments (foraminifera and other fauna), large fragments account for $22-100 \%$ of the material, with small fragments making up $0-88 \%$ of recovered specimens (Tables $1-3$ ).

Table 3. Comprehensive data on the Northwest Beris, Southeast Water Desalination, Crab Island and Park Estuary sites (samples C115 to C16-8) of the Iranian Makran coast, Oman Sea, including location, sediment, grains/size, foraminifera, and corrosion levels.

\begin{tabular}{|c|c|c|c|c|c|c|}
\hline Sample no. & C11-5 & $\mathrm{C} 12-8$ & C13-8 & C14-9 & C15-5 & C16-8 \\
\hline Location & NW Beris Estuary & $\begin{array}{l}\text { NW Beris } \\
\text { Estuary }\end{array}$ & NW Beris Estuary & $\begin{array}{c}\text { SE Water } \\
\text { Desalination }\end{array}$ & Crab Island & Park Estuary \\
\hline Coordinates & $\begin{array}{l}\text { N } 25^{\circ} .11^{\prime} .35 .3^{\prime \prime} \\
\text { E } 61^{\circ} .05^{\prime} .14 .0^{\prime \prime}\end{array}$ & $\begin{array}{l}\text { N } 25^{\circ} .11^{\prime} .46 .0^{\prime \prime} \\
\text { E } 61^{\circ} .04^{\prime} .24 .7^{\prime \prime}\end{array}$ & $\begin{array}{l}\text { N } 25^{\circ} .12^{\prime} .18 .6^{\prime \prime} \\
\text { E } 61^{\circ} .02^{\prime} .21 .6^{\prime \prime}\end{array}$ & $\begin{array}{l}\text { N } 25^{\circ} .26^{\prime} .18 .7^{\prime \prime} \\
\text { E } 60^{\circ} .29^{\prime} .22 .1^{\prime \prime}\end{array}$ & $\begin{array}{l}\text { N } 25^{\circ} .24^{\prime} .22 .3^{\prime \prime} \\
\text { E } 60^{\circ} .25^{\prime} .27 .6^{\prime \prime}\end{array}$ & $\begin{array}{l}\text { N } 25^{\circ} .25^{\prime} .07 .0^{\prime \prime} \\
\text { E } 60^{\circ} .35^{\prime} .44 .1^{\prime \prime}\end{array}$ \\
\hline Environment & $\begin{array}{c}\text { Supratidal, Tidal } \\
\text { channel Levee }\end{array}$ & $\begin{array}{c}\text { Supratidal, Tidal } \\
\text { channel Levee }\end{array}$ & $\begin{array}{l}\text { Intertidal, Tidal } \\
\text { channel levee }\end{array}$ & Subtidal & $\begin{array}{c}\text { Subtidal, Tidal } \\
\text { channel levee }\end{array}$ & $\begin{array}{c}\text { Intertidal, Tidal } \\
\text { channel levee }\end{array}$ \\
\hline Sediments & Sand & Sand & Costal sand & Mud (clayey) & Silt, mud and sand & Costal sand \\
\hline Grains/Size & Fine to coarse sand & $\begin{array}{c}\text { Fine to coarse } \\
\text { sand }\end{array}$ & Fine to coarse sand & Mud, fine grains & $\begin{array}{l}\text { Mud, silt \& fine to } \\
\text { coarse sand }\end{array}$ & $\begin{array}{c}\text { Very fine to very } \\
\text { coarse sand }\end{array}$ \\
\hline Sorting & Fairly well sorted & $\begin{array}{c}\text { Fairly well } \\
\text { sorted }\end{array}$ & Medium sorted & Fairly well sorted & Poorly sorted & Medium sorted \\
\hline Foraminifera & $\begin{array}{c}\text { (99.3\%) } \\
\text { Ammonia convexa } \\
\text { Ammonia aoteana } \\
\text { Assilina } \\
\text { ammonoides } \\
\text { Cassidelina sp. } \\
\text { Elphidium } \\
\text { craticulatum } \\
\text { Heterolepa } \\
\text { subhaidingeri } \\
\text { Laticarinina } \mathrm{sp} . \\
\text { Lenticulina imbosa } \\
\text { Pararotalia } \\
\text { nipponica } \\
\text { Miliolids }\end{array}$ & $\begin{array}{c}(94 \%) \\
\text { Ammonia sp. } \\
\text { Ammonia convexa } \\
\text { Ammonia } \\
\text { aoteana } \\
\text { Assilina } \\
\text { ammonoides } \\
\text { Bolivina } \mathrm{sp} . \\
\text { Cassidelina? } \mathrm{sp} . \\
\text { Elphidium } \mathrm{sp} . \\
\text { Elphidium } \\
\text { craticulatum } \\
\text { Lenticulina } \mathrm{sp} . \\
\text { Lenticulina } \\
\text { imbosa } \\
\text { Lenticulina } \\
\text { cultrate } \\
\text { Siphogenerina } \\
\text { sp. } \\
\text { Textularia } \mathrm{sp} . \\
\text { Miliolids }\end{array}$ & $\begin{array}{c}\text { (96.7\%) } \\
\text { Ammonia convexa } \\
\text { Assilina sp. } \\
\text { Assilina } \\
\text { ammonoides } \\
\text { Asterorotalia } \\
\text { gaimardi } \\
\text { Bovilina striatula } \\
\text { Elphidium } \\
\text { craticulatum } \\
\text { Elphidium } \\
\text { limbatum } \\
\text { Globigerinoides } \\
\text { sp. } \\
\text { Heterolepa } \\
\text { subhaidingeri } \\
\text { Lenticulina } \\
\text { cultrate } \\
\text { Lenticulina imbosa } \\
\text { Pararotalia } \mathrm{sp} . \\
\text { Siphogenerina } \mathrm{sp} . \\
\text { Uvigerina schencki } \\
\text { Miliolids }\end{array}$ & $\begin{array}{c}\text { (97.8\%) } \\
\text { Ammonia convexa } \\
\text { Assilina } \\
\text { ammonoides } \\
\text { Asterorotalia } \\
\text { gaimardi } \\
\text { Asterorotalia } \\
\text { dentate } \\
\text { Elphidium } \\
\text { limbatum } \\
\text { Elphidium } \\
\text { craticulatum } \\
\text { Eponides? sp. } \\
\text { Globigerina } \\
\text { bulloides } \\
\text { Heterolepa } \\
\text { subhaidingeri } \\
\text { Lenticulina } \mathrm{sp} . \\
\text { Pararotalia } \mathrm{sp} . \\
\text { Pararotalia } \\
\text { nipponica } \\
\text { Spiroplectinella } \mathrm{sp} . \\
\text { Uvigerina schencki } \\
\text { Miliolids }\end{array}$ & $\begin{array}{c}\text { (97.8\%) } \\
\text { Ammonia } \mathrm{sp} . \\
\text { Ammonia convexa } \\
\text { Ammonia } \\
\text { parkinsoniana } \\
\text { Ammonia } \\
\text { takanabensis } \\
\text { Ammonia } \mathrm{cf} . \\
\text { aomoriensis } \\
\text { Assilina } \\
\text { ammonoides } \\
\text { Asterorotalia } \\
\text { gaimardi } \\
\text { Bolivina striatula } \\
\text { Cibicides } \mathrm{sp} ., \\
\text { Elphidium } \mathrm{sp} . \\
\text { Eponides? } \mathrm{sp} ., \\
\text { Lenticulina } \mathrm{sp} . \\
\text { Globigerinoides } \mathrm{sp} . \\
\text { Nodosaria } \text { sp., } \\
\text { Pararotalia } \mathrm{sp} . \\
\text { Pararotalia } \\
\text { nipponica } \\
\text { Pyramidulina } \mathrm{sp} . \\
\text { Spiroplectinella } \mathrm{sp} . \\
\text { Miliolids }\end{array}$ & $\begin{array}{c}\text { (94.4\%) } \\
\text { Ammonia convexa } \\
\text { Ammonia aoteana } \\
\text { Amphistegina sp. } \\
\text { Assilina ammonoides } \\
\text { Bolivlina } \text { sp., } \\
\text { Bolivina striatula } \\
\text { Elphidium } \\
\text { fichteliahum } \\
\text { Elphidium } \\
\text { craticulatum } \\
\text { Elphidium crispum } \\
\text { Globigerinoides } \\
\text { trilobus } \\
\text { Heterolepa } \\
\text { subhaidingeri } \\
\text { Lenticulina imbosa } \\
\text { Lenticulina cultrate } \\
\text { Laevidentalina } \text { sp. } \\
\text { Nonion subturgidum } \\
\text { Operculina } \text { sp., } \\
\text { Pararotalia } \text { sp. } \\
\text { Pararotalia nipponica } \\
\text { Uvigerina schencki, } \\
\text { Miliolids }\end{array}$ \\
\hline Other fauna & Ostracoda $(0.7 \%)$ & $\begin{array}{c}\text { Ostracoda } \\
(1.7 \%) \\
\text { Sponge }(1.7 \%) \\
\text { Gastrapoda } \\
(2.6 \%) \\
\end{array}$ & $\begin{array}{c}\text { Ostracoda }(2 \%) \\
\text { Sponge }(0.65 \%) \text {, } \\
\text { Gastrapoda } \\
(0.65 \%)\end{array}$ & $\begin{array}{c}\text { Gastrapoda } \\
(1.48 \%) \\
\text { Sponge }(0.72 \%)\end{array}$ & $\begin{array}{c}\text { Ostracoda }(0.56 \%) \\
\text { Bivalve }(0.54 \%) \\
\text { Sponge }(1.1 \%)\end{array}$ & $\begin{array}{c}\text { Ostracoda }(0.85 \%) \\
\text { Gastrapoda }(2.6 \%) \\
\text { Sponge }(2.15 \%)\end{array}$ \\
\hline $\begin{array}{c}\text { The most } \\
\text { abundant } \\
\text { fauna }\end{array}$ & $\begin{array}{c}\text { Ammonia convexa } \\
(42.7 \%) \\
\text { Elphidium } \\
\text { craticulatum }(13.8 \%) \\
\text { Miliolids }(11.7 \%)\end{array}$ & $\begin{array}{c}\text { Ammonia convexa } \\
(28 \%) \text { Ammonia } \\
\text { aoteana }(20 \%) \\
\text { Miliolids }(17.3 \%)\end{array}$ & $\begin{array}{c}\text { Ammonia convexa } \\
(16.32 \%) \\
\text { Assilina } \\
\text { ammonoides } \\
(21 \%) \\
\text { Elphidium } \\
\text { craticulatum }(25.8 \%) \\
\end{array}$ & $\begin{array}{c}\text { Ammonia convexa } \\
(15.2 \%) \\
\text { Assilina ammonoides } \\
(30.6 \%) \\
\text { Elphidium } \\
\text { craticulatum }(11.9 \%)\end{array}$ & $\begin{array}{c}\text { Ammonia convexa } \\
(15.2 \%) \\
\text { Spiroplectinella sp. } \\
(20.8 \%) \\
\text { Miliolids }(14.6 \%)\end{array}$ & $\begin{array}{c}\text { Ammonia convexa } \\
(40.7 \%) \\
\text { Assilina ammonoides } \\
(16.8 \%) \\
\text { Elphidium } \\
\text { craticulatum }(18 \%)\end{array}$ \\
\hline $\begin{array}{l}\text { Total number } \\
\text { of specimens }\end{array}$ & 260 & 204 & 219 & 141 & 234 & 401 \\
\hline $\begin{array}{c}\text { Forams with } \\
\text { iron stain }\end{array}$ & $78.6 \%$ & $31 \%$ & $49 \%$ & $21.6 \%$ & $31.4 \%$ & $59.7 \%$ \\
\hline $\begin{array}{c}\text { Max } \\
\text { Corrosion }\end{array}$ & $25 \%$ & $46 \%$ & $30 \%$ & $0 \%$ & $22 \%$ & $27 \%$ \\
\hline $\begin{array}{c}\text { Mod } \\
\text { Corrosion } \\
\end{array}$ & $42 \%$ & $23 \%$ & $20 \%$ & $0 \%$ & $56 \%$ & $27 \%$ \\
\hline $\begin{array}{c}\text { Minor } \\
\text { Corrosion }\end{array}$ & $33 \%$ & $70.3 \%$ & $50 \%$ & $100 \%$ & $22 \%$ & $46 \%$ \\
\hline $\begin{array}{c}\text { Large } \\
\text { fragments }\end{array}$ & $25.8 \%$ & $29.3 \%$ & $61.5 \%$ & $88.1 \%$ & $77 \%$ & $39 \%$ \\
\hline $\begin{array}{c}\text { Small } \\
\text { fragments }\end{array}$ & $74.2 \%$ & $70.3 \%$ & $38.5 \%$ & $11.9 \%$ & $23 \%$ & $61 \%$ \\
\hline
\end{tabular}



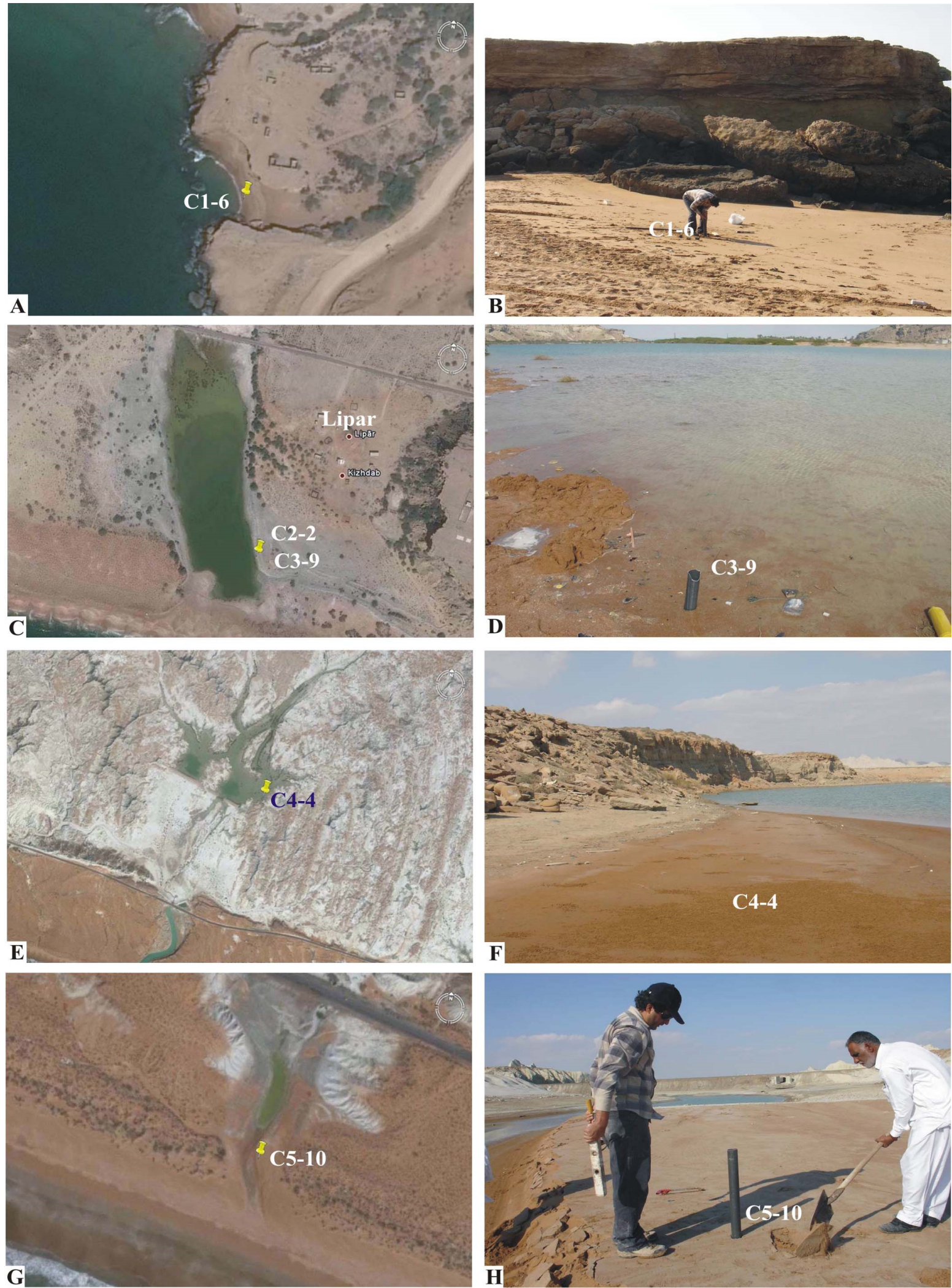

Figure 2. Study sites seen using Google Earth and in the field: A, B, Ramin Estuary (sample C1-6); C, D, Lipar Estuary (samples C2-2 and C3-9); E, F, Gareendar Estuary (sample C4-7); and G, H, Kochak Estuary (sample C5-10). 


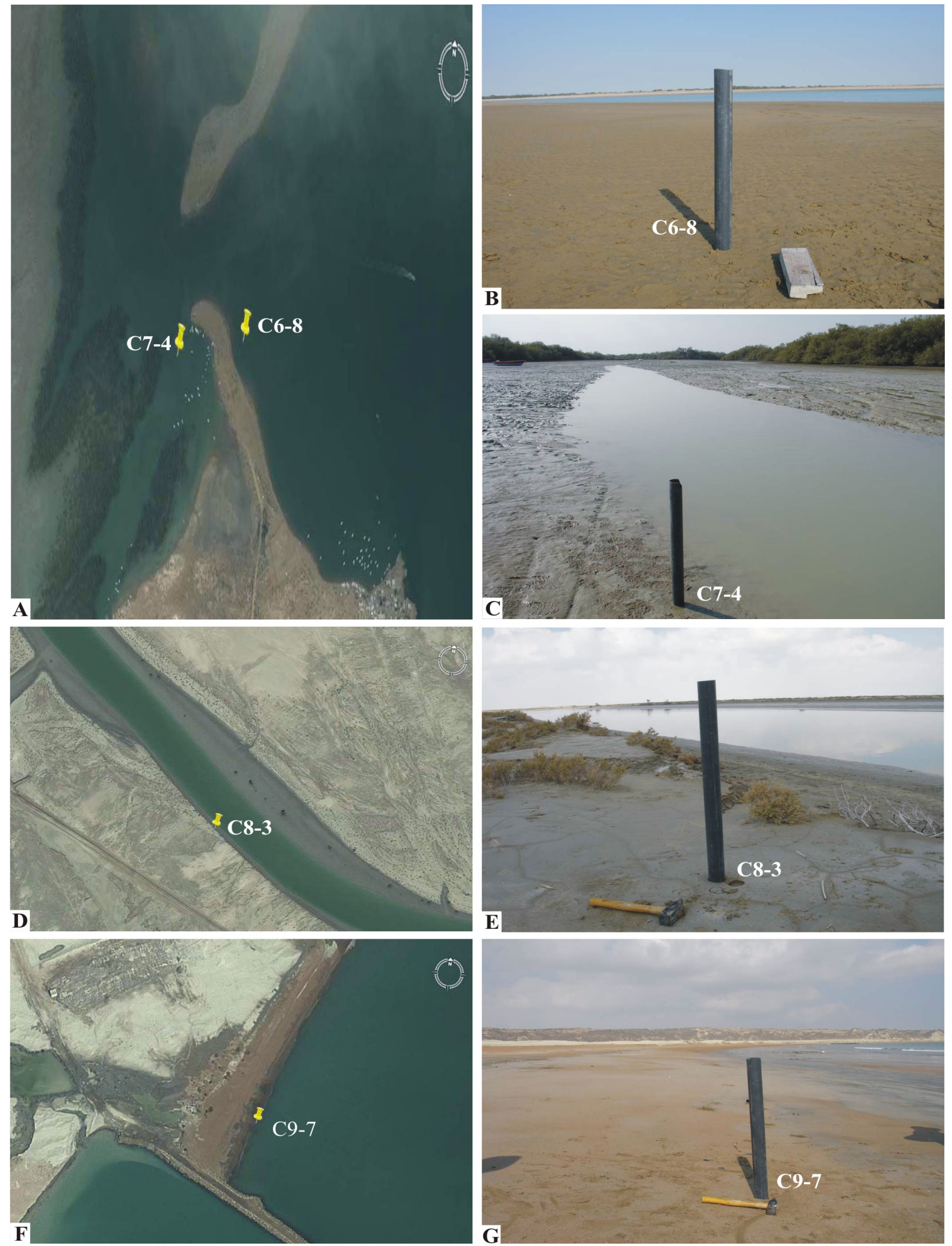

Figure 3. Study sites seen using Google Earth and in the field: A-E, Gawater Estuary (samples C6-8 to C8-3); and F, G, Pasabandar (sample C9-7). 

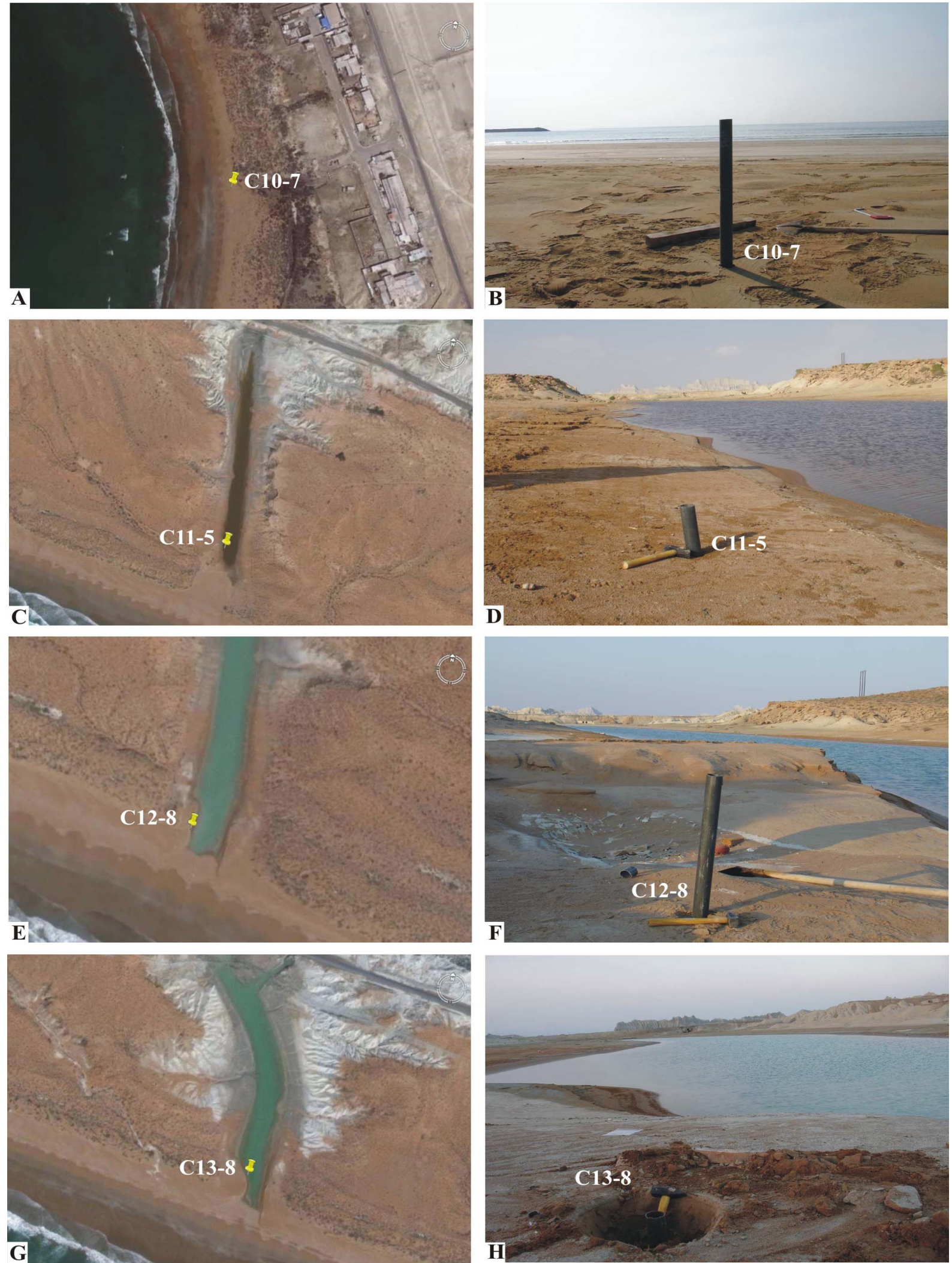

Figure 4. Study sites seen using Google Earth and in the field: A, B, Beris (sample C10-7); and C-H, Northwest Beris (samples C11-5 to $\mathrm{C} 13-8$ ) 

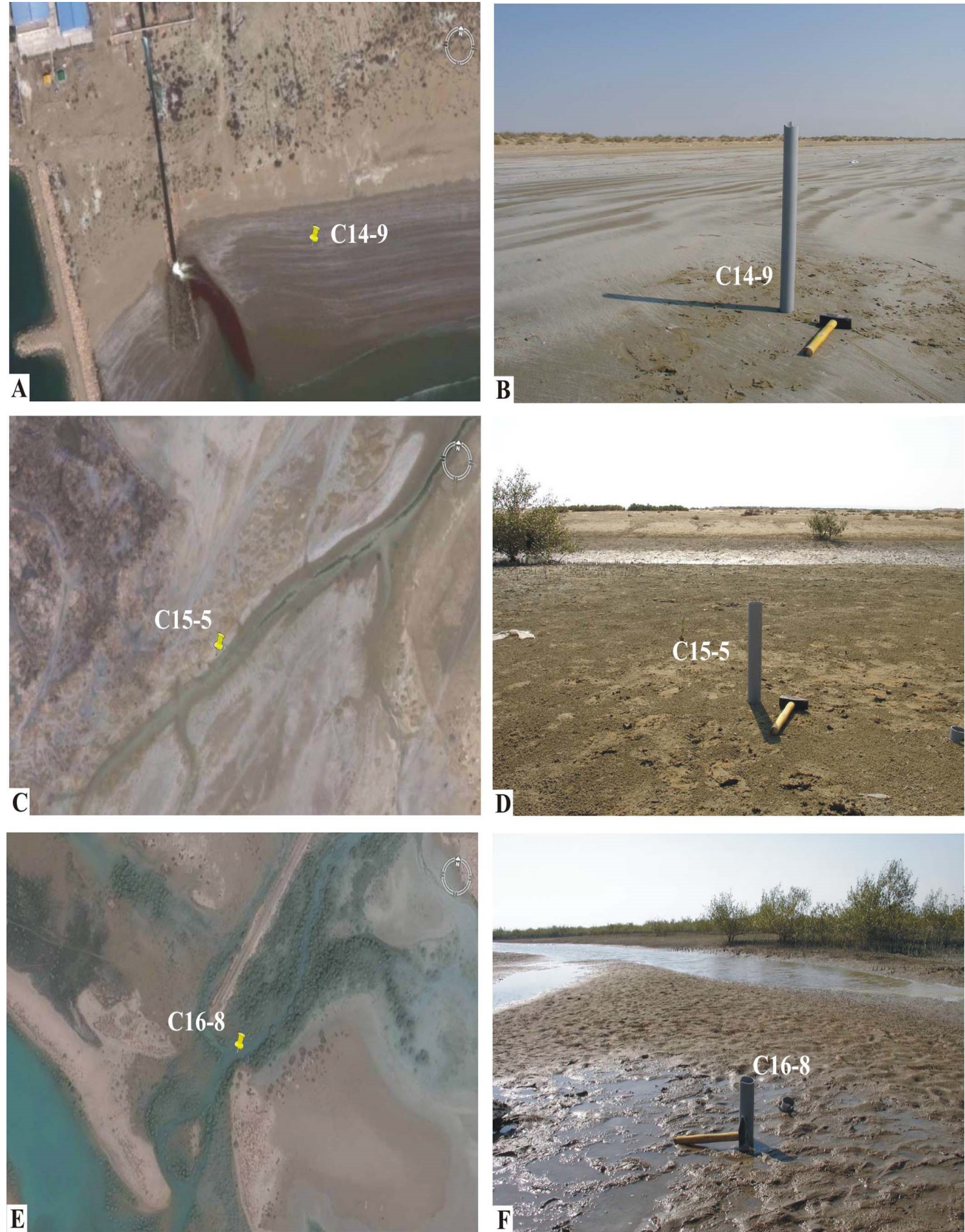

Figure 5. Study sites seen using Google Earth and in the field: A, B, Southeast Water Desalination (sample C14-7); C, D, Crab Island (sample C15-5); and E, F, Park Estuary (sample C16-8).

\section{Tidal channel subenvironment}

Tidal channels yielded sediments composed of medium to well sorted, fine to very coarse sand (Sample C2-2, C3-9, C4-4, C5-10, C10-7, C11-5, and C12-8). This sub-environment is predominantly supratidal in nature, but, like the mudflats, is also linked to tidal channel levee environments. The most abundant foraminifera in 
these samples are Ammonia convexa, Ammonia. aoteana, Assilina ammonoides, Elphidium craticulatum, Lenticulina sp., and various miliolids. Apart from the foraminifera, ostracods, gastropods and sponges are also recovered from these sites. Overall, $31-79 \%$ of foraminifera in tidal channel samples have iron stained tests, with maximum, moderate, and minor levels of corrosion recorded in a range of $0-53 \%, 19-47 \%$, and $26-80 \%$ of specimens, respectively. Among the shell fragments (foraminifera and other fauna), large fragments account for $26-62 \%$ of the material, with small fragments making up $37-74 \%$ of recovered specimens (Tables 1-3).

\section{Cluster analysis}

Partitioning Around Medoids (PAM) cluster analysis identified three biofacies within the study area (Fig. 12; tables 1-3) that have a collective average silhouette width of 0.68 : $\mathrm{BF}_{1}$ (intertidal environment; Average silhouette width $=0.82$ ), $\mathrm{BF}_{2}$ (supratidal environment; Average silhouette width $=0.65)$, and $\mathrm{BF}_{3}$ (subtidal environment; Average silhouette width $=0.63)$. $\mathrm{BF}_{1}$ is characterized by sandy beaches, high abundances of heavily corroded individuals (4760\%), and high abundances of Ammonia convexa, Assilina ammonoides, and Elphidium craticulatum (samples $\mathrm{C} 1-6, \mathrm{C} 9-7, \mathrm{C} 13-8$, and $\mathrm{C} 16-8$ ). $\mathrm{BF}_{2}$ is characterized by tidal channels, high abundances of iron-stained individuals (31-79\%), and high abundances of Ammonia aoteana, and Lenticulina sp. (samples C2-2, C3-9, C4-4, C5-10, C10-7, C11-5, and $\mathrm{C} 12-8) . \mathrm{BF}_{3}$ is characterized by mudflat environments (deltas and estuaries), low abundances of iron-stained individuals (14-36\%), high abundances of moderately corroded individuals and high abundances of the foraminifera Ammonia convexa, Asterorotalia galimardi, Elphidium craticulatum, Pararotalia nipponica, and Spiroplectinella sp. (samples C6-8, C7-4, C8-3, C14-9, and C15-5).

As mentioned above, samples collected from supratidal locations show an average of maximum iron staining and corrosion than samples collected from intertidal and subtidal locations. However, for all samples, a significant percentage of foraminifera (14-79\%) show iron staining and corrosion and as a whole, the range abundances of corroded specimens are $0-53 \%$ showing maximum corrosion, $0-56 \%$ having moderate corrosion and $17-100 \%$ showing minimum corrosion. Among the shell fragments, large fragments make up $26-100 \%$ of specimens, with small fragments forming $0-74 \%$ of the material.
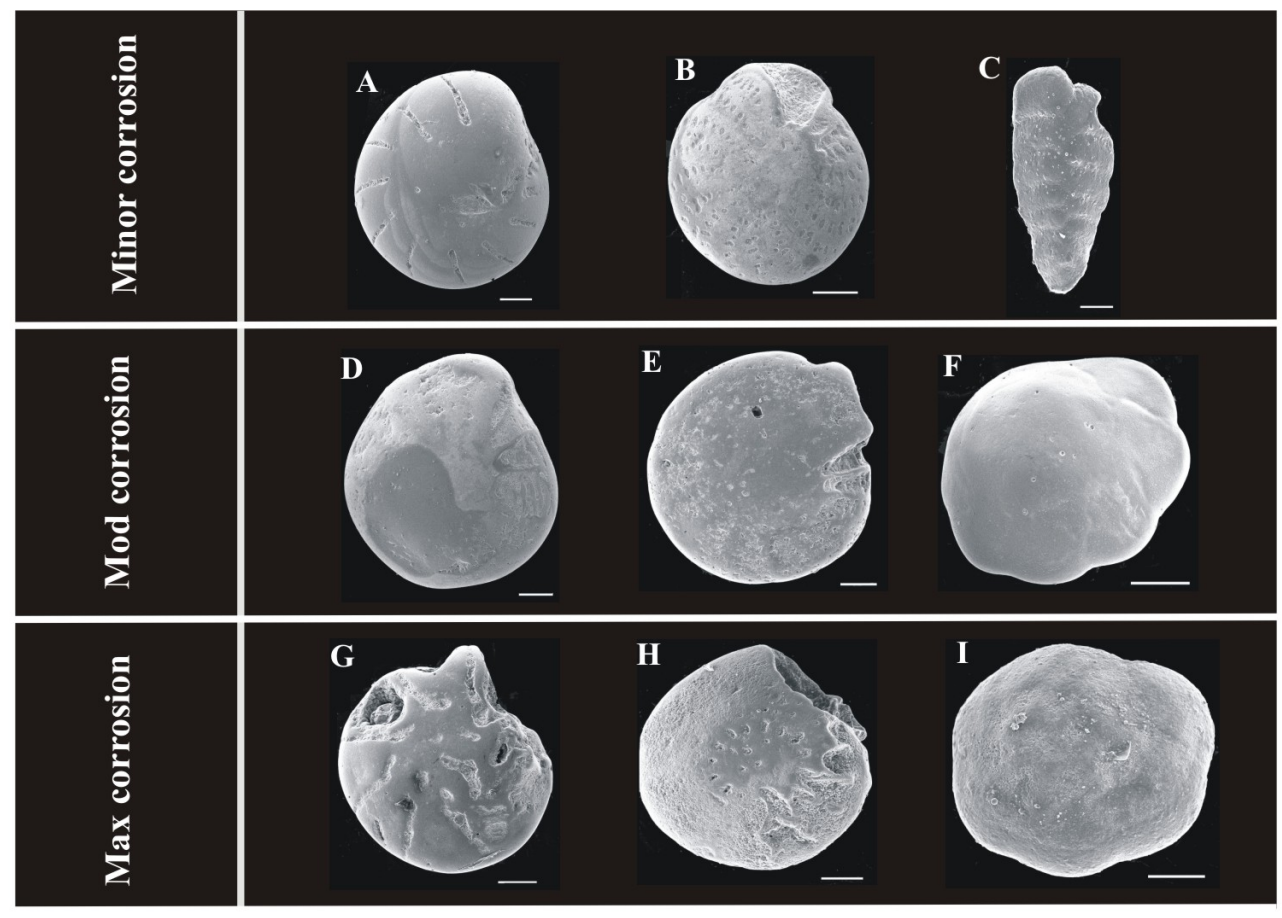

Figure 6. Examples of minimally (A-C), moderately (D-F), and maximally (G-I) corroded fossil foraminifera as defined in this paper. All scale bars represent $100 \mu \mathrm{m}$ 


\begin{tabular}{|c|c|c|c|c|c|c|c|c|c|c|c|c|c|c|c|c|c|c|c|c|c|c|c|c|c|c|c|c|c|c|}
\hline Species & \begin{tabular}{|l|}
\multicolumn{2}{|c|}{$\mathrm{C} 1$} \\
$\vec{\pi}$ \\
0 \\
0 \\
\end{tabular} & \begin{tabular}{l|}
-6 \\
30 \\
30 \\
\end{tabular} & 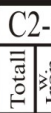 & & 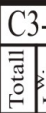 & & 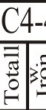 & & 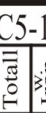 & & 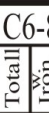 & & 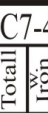 & & 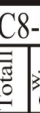 & & 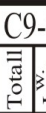 & $\begin{array}{l}-7 \\
0 \\
0 \\
0\end{array}$ & 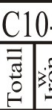 & $\begin{array}{l}0-7 \\
0: 0 \\
0\end{array}$ & C11- & $5 \mathrm{C}$ & $12-8$ & $\begin{array}{l}\mathrm{C} 13 \\
\\
\\
0\end{array}$ & $\begin{array}{l}3-8 \\
30 \\
30=0\end{array}$ & \begin{tabular}{l|l}
$\mathrm{C}$ \\
$\mathrm{C}$
\end{tabular} & \begin{tabular}{l|l|}
-9 & $\mathrm{Cl}$ \\
0.50 \\
0
\end{tabular} & $15-5$ & \begin{tabular}{|l|l}
\multicolumn{2}{|c|}{$\mathrm{C} 16$} \\
\multirow{2}{*}{}
\end{tabular} & $\begin{array}{l}6-8 \\
5=9 \\
=0\end{array}$ \\
\hline Ammonia sp. & & & & & & & & & & & & & & & & & & & & & & & $\%$ & & & & $9 \%$ & $\begin{array}{l}1.12 \\
\%\end{array}$ & & \\
\hline Ammonia convexa & $36 \%$ & $\begin{array}{rl}19.23 & 2 \\
0 & 0\end{array}$ & $\begin{array}{l}34.72 \\
\% \\
\%\end{array}$ & $\begin{array}{ll}28.22 \\
\%\end{array}$ & $23 \%$ & $\begin{array}{l}12.76 \\
\%\end{array}$ & $\%$ & $5 \% 5$ & $51 \% 3$ & $77 \%$ & 28.65 & $\%$ & \%2. & $\begin{array}{l}2.9 \\
\% \\
\end{array}$ & & & $\begin{array}{ll}14.4 \\
\% \\
0.4\end{array}$ & $\begin{array}{ll}5.2 \\
\% \\
\%\end{array}$ & $19 \%$ & $\begin{array}{l}10.8 \\
0.8 \\
\end{array}$ & & & & $\begin{array}{c}16.33 \\
\% \\
\%\end{array}$ & $\begin{array}{r}7.5 \\
\% \\
\end{array}$ & \%.7. & $\begin{array}{l}15.2 \\
\% \\
\%\end{array}$ & $\begin{array}{c}2.8 \\
\%\end{array}$ & $\begin{array}{r}40.7 \\
\%\end{array}$ & $\begin{array}{l}24 \\
\%\end{array}$ \\
\hline Ammonia aoteana & $5.7 \%$ & $5 \%$ & \begin{tabular}{l|l}
7.2 & 5.5 \\
0 & 5
\end{tabular} & $5.2 \%$ & $5.2 \%$ & & $3.9 \%$ & $9 \% 1$ & \begin{tabular}{l|l}
2.4 & 13 \\
$\%$ & \\
\end{tabular} & $\begin{array}{l}12.4 \\
\% \\
\end{array}$ & $1.3 \% 1$. & $3 \%$ & & & & & $\begin{array}{ll}1.7 \\
\%\end{array}$ & $\begin{array}{ll}.7 \\
\%\end{array}$ & $\begin{array}{ll}4.5 & 4 \\
\% & 4 \\
\end{array}$ & \begin{tabular}{l|l}
4.5 & 5 \\
$\%$ & \\
\end{tabular} & & $\begin{array}{l}0 \\
6 \\
6\end{array}$ & $720 \%$ & & & & & & \begin{tabular}{|r|r|}
2.7 & 1 \\
& \\
\end{tabular} & 1.8 \\
\hline Ammonia tepida & & & & & & & \begin{tabular}{l|l}
3.9 & 2 \\
$\%$ & 2 \\
\end{tabular} & $\begin{array}{ll}2.1 \\
\% \\
\end{array}$ & & & & & & & & & & & & & & & & & & & & & & \\
\hline Ammonia parkinsoniana & & & & & \begin{tabular}{|l|l|}
2.2 \\
$\%$ \\
\end{tabular} & \begin{tabular}{|l|}
2.2 \\
$\%$ \\
\end{tabular} & & & & & & & & & & & & & & & & & & & & & & & & \\
\hline Ammonia takanabensis & & & & & & & & & & & & & & & & & & & & & & & & & & & & & & \\
\hline Ammonia cf. aomoriensis & & & & & & & & & & & & & & & & & & & & & & & & & & & & & & \\
\hline Ammonia inflata & & & & & & & $\begin{array}{l}3.92 \\
\% \\
\end{array}$ & $\begin{array}{l}2.1 \\
\% \\
\end{array}$ & & & & & & & & & & & & & & & & & & & & & & \\
\hline Anomalinulla glabrata & $0.8 \%$ & $0.8 \%$ & & & & & & & & & & & & & & & & & & & & & & & & & & & & \\
\hline Amphistegina sp. & & & & & & & & & & & & & & & & & & & & & & & & & & & & & $\begin{array}{ll}0.9 \\
\% \\
\%\end{array}$ & $\begin{array}{c}0.9 \\
\%\end{array}$ \\
\hline Amphistegina lessonii & & & $\begin{array}{ll}3.2 \\
\% \\
\end{array}$ & $\begin{array}{ll}2.4 \\
\% \\
\end{array}$ & \begin{tabular}{|c|}
1.5 \\
$\%$ \\
\end{tabular} & \begin{tabular}{l|l|}
0.75 \\
$\%$
\end{tabular} & & & & & & & & & & & & & & & & & & & & & & & & \\
\hline Amphicoryna scalaris & & & & & & & & & & & & & & & & & $\begin{array}{c}0.75 \\
\% \\
\end{array}$ & & & & & & & & & & & & & \\
\hline Assilina? sp. & & & & & & & & & & & & & & & & & & & & & & & & $\begin{array}{c}0.68 \\
0\end{array}$ & 0.68 & & & & & \\
\hline Assilina ammonoides & $8.3 \%$ & $6.70 \%$ & $2.4 \% 1$. & $1.6 \%$ & $3 \%$ & $3 \%$ & $\begin{array}{ll}6.45 \\
\% \\
\%\end{array}$ & $\begin{array}{ll}5.74 \\
\% \\
\end{array}$ & 4.193 & $3.1 \%$ & 8.93 & $\begin{array}{l}3.81 \\
\% \\
3.1\end{array}$ & $\begin{array}{ll}10.12 \\
0.12\end{array}$ & 909 & $9 \%$ & $9 \% 2$ & $\begin{array}{ll}20.91 \\
\% 1\end{array}$ & $\begin{array}{ll}11.5 \\
0.5\end{array}$ & 18.21. & $\begin{array}{l}2.8 \\
\% \\
\end{array}$ & & 9 & \begin{tabular}{l|l}
2.7 & 1.8 \\
0 & $\%$
\end{tabular} & $\begin{array}{c}21.01 \\
\%\end{array}$ & $\begin{array}{c}10.2 \\
\% \\
\end{array}$ & $\begin{array}{ll}0.65 \\
\%\end{array}$ & \begin{tabular}{|l|l|}
2 & 11.8 \\
2 & 0 \\
\end{tabular} & $\begin{array}{c}4.5 \\
0 \\
0\end{array}$ & $\begin{array}{r}16.86 \\
0.6\end{array}$ & $\begin{array}{l}6.8 \\
8 \\
0\end{array}$ \\
\hline Asterorotalia gaimardi & & & & & & & & & $.3 \%$ & 0.257 & \begin{tabular}{c|c|c|}
$7 \%$ & 1 \\
\end{tabular} & 1.31 & $3 \% 1.4$ & 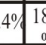 & $\begin{array}{ll}8.2 \\
0.2\end{array}$ & & 6.35 & & $\begin{array}{ll}1.2 \\
0\end{array}$ & 1.2 & & & & 0.68 & & \begin{tabular}{l|l}
1. \\
$9 \%$
\end{tabular} & .51 .12 & & & \\
\hline Asterorotalia dentata & & & & & & & & & & & & & & & & & & & & & & & & & & 0.75 & $\begin{array}{r}1.7 \\
\% \\
\end{array}$ & & & \\
\hline Bolivina sp. & & & & & & & & & & & & & & & & & & & $2 \% 0$ & 40. & & & 90.9 & $\begin{array}{c}0.680 \\
0\end{array}$ & $\begin{array}{c}0.68 \\
\% \\
\end{array}$ & & & & $\begin{array}{l}1.8 \\
\% \\
\%\end{array}$ & $\begin{array}{l}0.9 \\
\%\end{array}$ \\
\hline Bolivina striatula & $1.7 \%$ & $0.8 \%$ & $12 \%$ & 8.907 & & & $\begin{array}{ll}1.8 & 1 . \\
\% & 0\end{array}$ & $\begin{array}{ll}8 & 11 \\
\% & 1\end{array}$ & $.3 \%$ & $\begin{array}{c}0.77 \\
\% \\
\end{array}$ & & & & & & & & & $\begin{array}{ll}2.1 \\
\% \\
\%\end{array}$ & $2 \%$ & & & & & & & $\begin{array}{l}1.12 \\
\% \\
\end{array}$ & & ${ }_{0}^{0.9}{ }^{0}$ & $\begin{array}{c}0.45 \\
\% \\
\end{array}$ \\
\hline Bolivina spathulata & & & & & \begin{tabular}{|c|}
0.75 \\
0 \\
$\%$
\end{tabular} & & & & $1 \% 0$ & $\begin{array}{l}0.77 \\
\% \\
\end{array}$ & & & & & $9 \%$ & & $\begin{array}{l}1.150 \\
0.4 \\
\end{array}$ & $\begin{array}{l}0.57 \\
0.7 \\
\end{array}$ & & & & & & & & & & & & \\
\hline Cassidelina sp. & & & & & & & & & & & & & $\begin{array}{l}0.48 \\
\% \\
\end{array}$ & & & & & & & & & $\begin{array}{l}1.7 \\
\% \\
\end{array}$ & $\begin{array}{lll}90.90 \\
\end{array}$ & & & & & & & \\
\hline Challengerella $\mathrm{sp}$. & & & & & & & $\begin{array}{l}5.7 \\
\\
\end{array}$ & & & & & & & & & & & & & & & & & & & & & & & \\
\hline Cibicides sp. & & & & & & & & & & & & & & & & & & & & & & & & & & $9 \%$ & $\begin{array}{l}.56 \\
\% \\
\end{array}$ & & & \\
\hline Cibicides pseudolobatulus & & & & & & & & & & & & & & & & & & & & $\begin{array}{l}1.2 \\
\% \\
\end{array}$ & & & & & & & & & & \\
\hline Cancris auriculus & & & & & & & & & & & $\begin{array}{l}1.63 \\
0 \\
0\end{array}$ & $\begin{array}{l}630 \\
60^{6} \\
\end{array}$ & $\begin{array}{ll}0.48 \\
0.4 \\
\end{array}$ & & & & & & & & & & & & & & & & & \\
\hline Cancris sagrum & & & & & & & & & & & $\begin{array}{l}1.63 \\
\% \\
\end{array}$ & & & & & & & & & & & & & & & & & & & \\
\hline Discorbinella rodiensis & & & & & & & & & & & & & & & & & $\begin{array}{c}0.57 \\
\% \\
\end{array}$ & $\begin{array}{ll}0.57 \\
\% \\
\end{array}$ & \begin{tabular}{l|l}
0.4 & 0 \\
$\%$ & 0 \\
\end{tabular} & $\begin{array}{l}0.4 \\
\% \\
\end{array}$ & & & & & & & & & & \\
\hline Elphidium sp. & & & & & & & & & & & & & & & & & $\begin{array}{ll}2.9 \\
\% \\
\end{array}$ & $\begin{array}{ll}1.7 \\
\%\end{array}$ & & & & & $\begin{array}{ll}2.7 \\
7 \\
0\end{array}$ & & & & $\begin{array}{r}3.4 \\
\% \\
\end{array}$ & $\begin{array}{r}1.7 \\
\% \\
\end{array}$ & \begin{tabular}{|c|c|}
2.3 & 1 \\
$\%$ & \\
\end{tabular} & $\begin{array}{l}1.8 \\
\% \\
\end{array}$ \\
\hline Elphidium excavatum & & & & & & & & & & & $\begin{array}{r}2.5 \\
\% \\
\end{array}$ & & & & & & $\begin{array}{c}1.15 \\
\% \\
\end{array}$ & & & & & & & & & & & & & \\
\hline Elphidium fichtelianum & & & & & & & & & & & $\begin{array}{l}0.63 \\
\% \\
\end{array}$ & & & & & & & & & & & & & & & & & & \begin{tabular}{|c|}
0.45 \\
$\%$ \\
\end{tabular} & \\
\hline Elphidium maorium & & & & & & & & & & & & & & & & & & & \begin{tabular}{l|l}
1.2 \\
$\%$ \\
0
\end{tabular} & $\begin{array}{l}0.4 \\
\% \\
\end{array}$ & & & & & & & & & & \\
\hline Elphidium crispum & $6.7 \%$ & $3.3 \%$ & $1.6 \%$ & $1.6 \%$ & $3 \%$ & \begin{tabular}{|l|}
2.2 \\
$\%$ \\
\end{tabular} & & & $0.5 \%$ & $\begin{array}{ll}0.25 \\
\%\end{array}$ & & & $\begin{array}{l}0.48 \\
\% \\
\end{array}$ & & & & $\begin{array}{l}1.15 \\
\% \\
\end{array}$ & $\begin{array}{c}0.57 \\
\% \\
\end{array}$ & & & & & & $\begin{array}{r}4.1 \\
\% \\
\end{array}$ & $\begin{array}{l}4.1 \\
\% \\
\end{array}$ & & & & $\begin{array}{r}1.8 \\
\% \\
\end{array}$ & \\
\hline Elphidium craticulatum & & & $\begin{array}{l}17.71 \\
\% \\
\end{array}$ & \begin{tabular}{|l|l|}
$12 \%$ \\
\end{tabular} & \begin{tabular}{|c|}
8.2 \\
$\%$ \\
\end{tabular} & $6 \% 1$ & $\begin{array}{ll}10.3 \\
\% \\
\end{array}$ & \begin{tabular}{l|l|}
7.8 \\
$\%$ \\
\end{tabular} & \begin{tabular}{l|l|l|l|l|}
2.2 & \\
\end{tabular} & \begin{tabular}{l|l}
8.5 & 1 \\
$\%$ & \\
\end{tabular} & $\begin{array}{ll}14.6 & 1 \\
\% & \\
\end{array}$ & \begin{tabular}{l|l}
1.9 \\
$\%$
\end{tabular} & \begin{tabular}{|l|l|}
12.5 & 0. \\
$\%$ & 0 \\
\end{tabular} & $\begin{array}{l}4.48 \\
\% \\
\end{array}$ & & & \begin{tabular}{r|r|}
10.4 \\
$\%$
\end{tabular} & \begin{tabular}{l|l}
3.5 & 1 \\
$\%$ & \\
\end{tabular} & $\begin{array}{r}12.5 \\
\% \\
\end{array}$ & \begin{tabular}{l|l|}
7.5 & 1. \\
$\%$ & \\
\end{tabular} & & & \begin{tabular}{l|l}
$0 \%$ & 7.3 \\
$\%$
\end{tabular} & \begin{tabular}{|r|r|}
25.8 & 5. \\
\end{tabular} & $5.4 \%$ & \begin{tabular}{rr|r}
11.9 & $60 \%$ \\
\end{tabular} & & & $18 \% 7$ & \begin{tabular}{|l|}
7.7 \\
$\%$ \\
\end{tabular} \\
\hline Elphidium limbatum & & & & & & & & & & & & & & & & & & & & & & & & $\begin{array}{l}0.68 \\
\% \\
\end{array}$ & & 0.75 & & & & \\
\hline Elphidium albanii & & & & & & & & & & & & & $\begin{array}{l}3.9 \\
\% \\
\end{array}$ & $\begin{array}{l}4.48 \\
\% \\
\end{array}$ & & & & & & & & & & & & & & & & \\
\hline Elphidium macellum & $\begin{array}{r}15.8 \\
\% \\
\end{array}$ & \begin{tabular}{l|}
5.8 \\
$\%$
\end{tabular} & & & & & & & & & & & & & & & & & & & & & & & & & & & & \\
\hline Eponides repandus & & & & & & & & & & & \begin{tabular}{l|l}
1.9 & 1 \\
$\%$ & \\
\end{tabular} & \begin{tabular}{l|}
.9 \\
$\%$ \\
\end{tabular} & & & & & $\begin{array}{l}2.9 \\
\% \\
\end{array}$ & $\begin{array}{l}2.9 \\
\% \\
\end{array}$ & & & & & & & & & $\begin{array}{l}1.12 \\
\% \\
\end{array}$ & $\begin{array}{c}0.56 \\
\% \\
\end{array}$ & & \\
\hline Globigerinoides sp. & $0.8 \%$ & & & & & & & & $\begin{array}{ll}.25 & 0 \\
\% & 0 \\
\end{array}$ & $\begin{array}{ll}0.25 \\
\% \\
\end{array}$ & \begin{tabular}{l|l}
1.3 & 1 \\
$\%$ & 1 \\
\end{tabular} & $\frac{3}{3} 0^{0}$ & $\begin{array}{l}0.48 \\
\% \\
\end{array}$ & & & & & & & & & & & \begin{tabular}{|c|c|}
0.68 & 0 \\
$\%$
\end{tabular} & $\begin{array}{c}0.68 \\
\% \\
\end{array}$ & & $\begin{array}{r}3.9 \\
\% \\
\end{array}$ & $\begin{array}{r}3.9 \\
\% \\
\end{array}$ & & \\
\hline Globigerinoides trilobus & & & & & & & $\%$ & $\begin{array}{l}0.7 \\
\% \\
\end{array}$ & & & & & & & & & & & & & & & & & & & & & & \begin{tabular}{|l|}
1.8 \\
$\%$ \\
\end{tabular} \\
\hline Globigerinoides ruber & & & & & & & & & & & & & & & & & $\begin{array}{l}4.6 \\
\% \\
\end{array}$ & $\begin{array}{r}2.3 \\
\% \\
\end{array}$ & & & & & & & & & & & & \\
\hline Globigerinita sp. & & & $2.4 \% 2$ & $2.4 \%$ & & & & & & & & & & & & & & & & & & & & & & & & & & \\
\hline Globorotalia sp. & & & & & $\begin{array}{r}0.75 \\
\% \\
\end{array}$ & $\begin{array}{r}0.75 \\
\% \\
\end{array}$ & & & & & & & & & & & & & & & & & & & & & & & & \\
\hline
\end{tabular}

Figure 7. Frequency percentage of foraminifera (A-G) in the studied samples. 


\begin{tabular}{|c|c|c|c|c|c|c|c|c|c|c|c|c|c|c|c|c|c|c|c|c|c|c|c|c|c|c|}
\hline$\frac{\text { Species }}{\text { Sample nu0. }}$ & \multicolumn{2}{|c|}{ 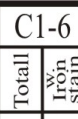 } & C2-2 & \multicolumn{2}{|c|}{ 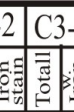 } & 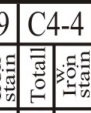 & \multicolumn{2}{|c|}{ 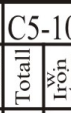 } & 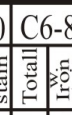 & \multicolumn{2}{|c|}{ 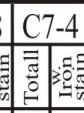 } & \multicolumn{2}{|c|}{ 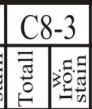 } & $\begin{array}{l}\text { C9-7 } \\
\text { 馬: }\end{array}$ & \multicolumn{2}{|c|}{ 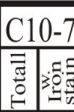 } & \multicolumn{2}{|c|}{ 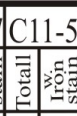 } & 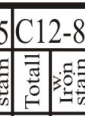 & \multicolumn{2}{|c|}{ 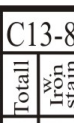 } & \begin{tabular}{|l|l|}
$\mathrm{C} 14$ \\
$\bar{a}$ \\
0
\end{tabular} & & 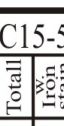 & 年16 & $16-8$ \\
\hline Globigerinella sp. & & & & & & & & & & & & & & 0.57 & & & & & & & & & & & & \\
\hline Globigerina bulloides & & & & & & & & & & & & & & & & & & & & & & & & & & \\
\hline Globoturborotalita? sp. & & & & & & & & & & & & & & & & $0.4 \%$ & & & & & & & & & & \\
\hline Gyroidina sp. & $0.8 \%$ & $0.8 \%$ & & & & & & & & & & & & & & & & & & & & & & & & \\
\hline Heterolepa subhaidingeri & & & & & & & & & $3 \%$ & $\begin{array}{c}0.48 \\
\%\end{array}$ & & & & & & & & & & & & $\begin{array}{c}0.75 \\
\%\end{array}$ & & & & \\
\hline Lobatula lobatula & & & & & & & & & & & & & & 1.15 & & & & & & & & & & & & \\
\hline Laevidentalina sp. & & & & & & & & & 50.630. & & & & & & & & & & & & & & & & $\begin{array}{l}1.8 \\
\% \\
\%\end{array}$ & \\
\hline Laevidentalia subsoluta & & & & & & 0.3. & & & & & & & & & & & & & & & & & & & & \\
\hline Lagena sp. & & & $3.2 \%$ & & & & & & & & & & & & & & & & & & & & & & & \\
\hline Laticarinina sp. & & & & & & & & & & & & & & & & & & & & & & & & & & \\
\hline Lenticulina & & & & & & & & & & & & & & & & & & & & & & $\begin{array}{r}0.77 \\
\% \\
\end{array}$ & & & & \\
\hline Lenticl & & & & & & & & & & & & & & & & & & & & & & & & & & \\
\hline Lenticl & & & & & & 0.70 & & 0.77 & & & & & & & & & & & 90 & & & & & & & (0.4) \\
\hline Lenticulina to & & $0.8 \%$ & & & & & & & & & & & & & & & & & & & & & & & & \\
\hline Lenticulina & & & $4 \% 3$ & $2.2 \% 8.5$ & & 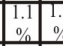 & & $3 \%$ & & 2.9 & \begin{tabular}{|l|}
1.9 \\
0.9
\end{tabular} & & & $0.5 \sqrt{0.0}$ & & & & & & & 8.68 & & & & & \\
\hline Loxostomin & & & & & & & & & & & & & & & & & & & & & & & & & & \\
\hline Miliol & & & & & & 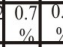 & & $\begin{array}{l}6.5 \\
6.1 \\
\%\end{array}$ & 210.8. & $190 \begin{array}{l}3.4 \\
0.4\end{array}$ & & $9 \%$ & & $19 \% 8$. & & & $\begin{array}{l}11.1 \\
\%\end{array}$ & $\%$ & & & & \begin{tabular}{|l|}
9.7 \\
0.9
\end{tabular} & & $\begin{array}{r}14.6 \\
\% \\
\end{array}$ & & 4 \\
\hline Monspeliensina $\mathrm{sp}$. & & & & & & & & & & & & & & & & & & & & & & & & & & \\
\hline Neocassidulina abbreviata & & & & & & & & & & & & & & & & & & & & & & & & & & \\
\hline Nodosaria sp. & & & $0.8 \%$ & & & 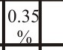 & & & & & & & & & & & & & & & & & & $\begin{array}{r}0.56 \\
\% \\
\end{array}$ & & \\
\hline Nonion sp. & & & & & & & & & & & & & & $\begin{array}{r}0.5 \\
\% \\
\%\end{array}$ & & & & & & & & & & & & \\
\hline Nonio & & & & & & & & & & & & & & $\begin{array}{l}1.15 \\
\% \\
\%\end{array}$ & & & & & & & & & & & & \\
\hline Opercu & & & & & & & & & & & & & & & & & & & & & & & & & & \\
\hline Pararotalia $\mathrm{sp}$. & 11.7 & & $0.8 \%$ & $\begin{array}{l}0.7 \\
\%\end{array}$ & & $\begin{array}{ll}0.7 \\
\% \\
\end{array}$ & $\begin{array}{ll}4.1 \\
\%\end{array}$ & $\begin{array}{l}1.8 .8 \\
0.4\end{array}$ & 50.50 & $\frac{63}{6}$ & \begin{tabular}{|l|l|l|l|l|}
0.48 \\
$\%$
\end{tabular} & $9 \%$ & 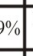 & $\begin{array}{l}1.15 \\
\% \\
\%\end{array}$ & $\%$ & \begin{tabular}{|l|}
4.1 \\
$\%$
\end{tabular} & & & & & & $\begin{array}{c}7.5 \\
0.5 \\
\end{array}$ & . & 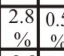 & & \\
\hline Pararotalia nipponica & & & & & & & $\frac{0.7}{\%}$ & $\begin{array}{l}3.1 \\
\% \\
\%\end{array}$ & & $\begin{array}{c}3.4 \\
\% \\
\%\end{array}$ & $\begin{array}{l}0.48 \\
\% \\
\end{array}$ & $\begin{array}{l}36.4 \\
\% \\
\end{array}$ & $9 \%$ & & 0.4 & & \begin{tabular}{|c|}
1.4 \\
$\%$ \\
\end{tabular} & & & & & \begin{tabular}{|l|}
1.5 \\
$\%$ \\
\end{tabular} & & $\begin{array}{ll}3.90 .5 \\
\%\end{array}$ & & $\begin{array}{ll}1: \\
0 \\
0.4\end{array}$ \\
\hline Perneroplis pertusus & & & $1.0^{\circ} / 1$ & .6\% & & & & & $1.3 \%$ & & & & & & & & & & & . & & & & & & \\
\hline Peneroplis planatus & & & & & & & & & & & & $9 \%$ & $9 \%$ & & & & & & & & & & & & & \\
\hline Pyramidulina sp. & & & & & & & & & & & & & & & & & & & & & & & & $\begin{array}{l}1.12 \\
\% \\
\end{array}$ & & \\
\hline Rosalina bradyi & & & & & & & & & & & & & & $\begin{array}{ll}1.14 & 1 . \\
\% & 1 . \\
\end{array}$ & & & & & & & & & & & & \\
\hline Rosalina orientalis & & & & & & & & & & & & & & & & & & & & & & & & & & \\
\hline Siphogenerina sp. & & & $0.8 \%$ & & & & & & & & & & & $\begin{array}{l}1.15 \\
\% \\
\%\end{array}$ & & & & & & & 60.6 & & & & & \\
\hline Siphogenerina raphana & & & & & & & & & & & & & & & & & & & & & & & & & & \\
\hline Siphotextularia heterastoma & & & & & & & & & & & & & & & & & & & & & & & & & & \\
\hline Spiroplectinella sp. & & & & & & & & & & & & & & & & & & & & & & $\begin{array}{c}6.7 \\
0\end{array} \mid$ & \% & & & ? \\
\hline Textularia $\mathrm{sp}$. & & & & & & & \begin{tabular}{l|l}
1.35 .1 \\
$\%$ \\
$\%$
\end{tabular} & $\begin{array}{l}.15 . \\
\% \\
0\end{array}$ & & $\begin{array}{l}1.9 \\
\%\end{array}$ & & & & & & $0.4 \%$ & & & & & & & & & $\begin{array}{l}0.45 \\
\% \\
\end{array}$ & 5 \\
\hline Uvigerina schencki & & & & $\%$ & $\begin{array}{l}5.7 \\
\% \\
\end{array}$ & & & & & & & & & & & & & & & 4 & $8 \begin{array}{l}8.8 \\
\%\end{array}$ & \begin{tabular}{|l|}
1.5 \\
$\%$ \\
\end{tabular} & & & & 150.4 \\
\hline Virgulinella sp. & & & & $\begin{array}{l}0.7 \\
\% \\
\%\end{array}$ & & & & & & & & & & & & & & & & & & & & & & \\
\hline Foraminifera & & & $\%$ & & 4 & $\begin{array}{ll}99.36 \\
\%\end{array}$ & $\begin{array}{l}100 \\
\%\end{array}$ & 0 & $\%$ & $\%$ & $\begin{array}{l}14 \\
\% \\
\end{array}$ & $\%$ & $\%$ & $\begin{array}{l}97.74 \\
\% \\
\% \\
0\end{array}$ & 59 & $\%$ & $\begin{array}{c}99.37 \\
\%\end{array}$ & & & 98 & 0 & . & $\stackrel{\circ}{\circ}$ & & & 459. \\
\hline Ostracoda & \begin{tabular}{|l}
1.6 \\
$\%$
\end{tabular} & & & & & & & & $\begin{array}{ll}10.8 \\
0 \%\end{array}$ & & & $\begin{array}{c}15.4 \\
\% \\
\end{array}$ & & & & \begin{tabular}{|c|}
0.8 \\
$\%$
\end{tabular} & & & 1.7 & 2 & $\%$ & 5 & & $\begin{array}{c}0.54 \\
\% \\
\end{array}$ & $\begin{array}{c}0.85 \\
\% \\
\end{array}$ & 35 \\
\hline Gastrapoda & & & & $\begin{array}{l}7.5 \\
0.7\end{array}$ & & $\begin{array}{ll}0.7 \\
\%\end{array}$ & & & $\begin{array}{l}1.5 \\
\% \\
\end{array}$ & $\begin{array}{l}0.5 \\
\% \\
\end{array}$ & & & & $\begin{array}{l}0.6 \\
\% \\
\end{array}$ & 0. & & \begin{tabular}{|l|}
0.7 \\
$\%$ \\
\end{tabular} & & $\%$ & & & \begin{tabular}{|c|}
1.48 \\
$\%$ \\
\end{tabular} & & & & 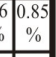 \\
\hline \begin{tabular}{|l|} 
Sponge \\
\end{tabular} & & & & $\frac{2.6}{\%}$ & & & & & $\begin{array}{l}2.7 \\
\% \\
\end{array}$ & & & & & $\begin{array}{l}1.7 \\
\% \\
\end{array}$ & & \begin{tabular}{|c|}
0.8 \\
$\%$ \\
\end{tabular} & & & $\%$ & 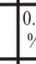 & & \begin{tabular}{|c|}
0.72 \\
$\%$ \\
\end{tabular} & & \begin{tabular}{|l|l}
1 \\
$\%$ \\
\end{tabular} & $\begin{array}{r}2.15 \\
\% \\
\end{array}$ & 15 \\
\hline Bivalve & & & & & & & & & & & & & & & & & & & & & & & & $\begin{array}{l}0.54 \\
0.6\end{array}$ & & \\
\hline
\end{tabular}

Figure 8. Frequency percentage of foraminifera $(\mathrm{G}-\mathrm{V})$, ostracods, gastropods, sponges and bivalves in the studied samples. 

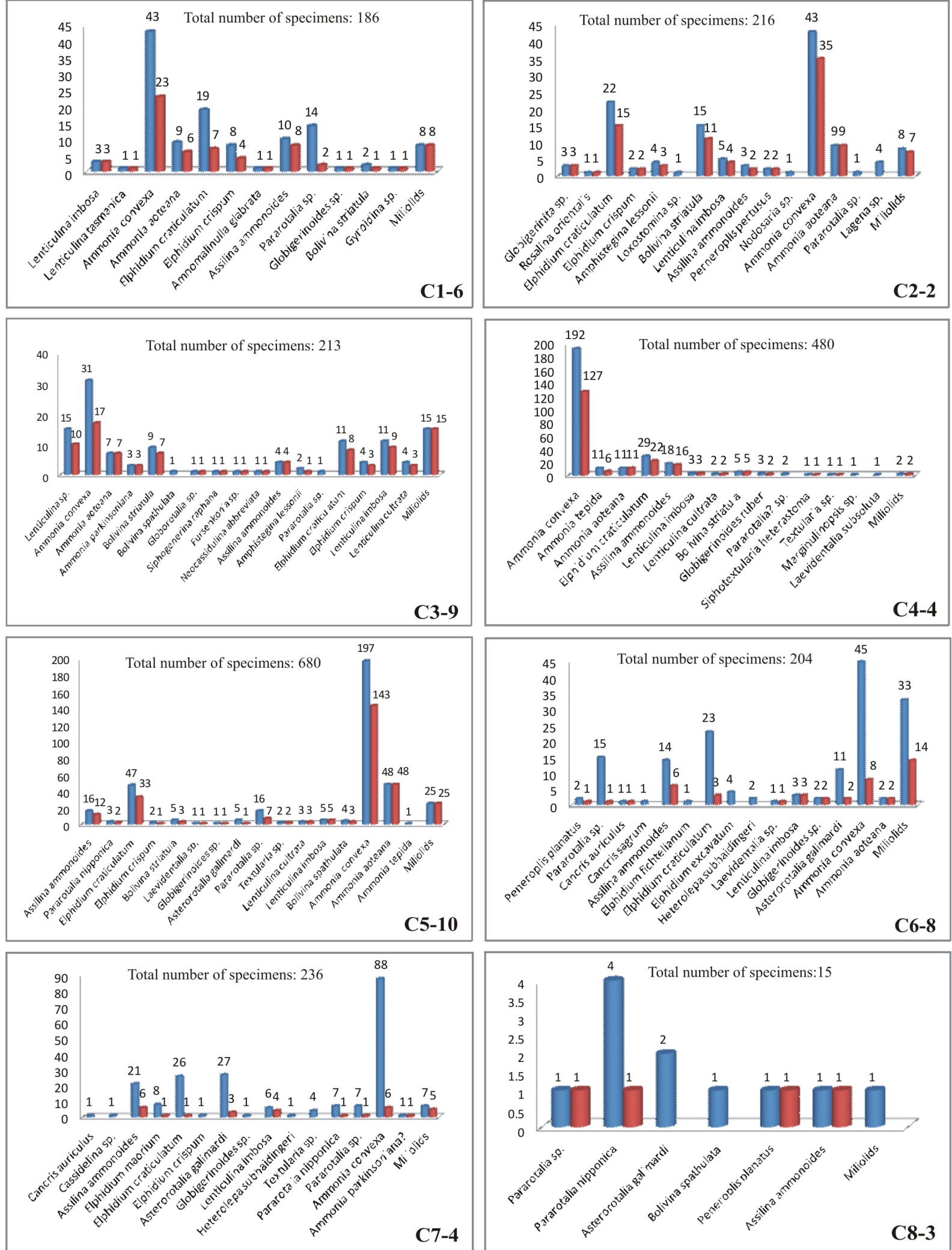

Figure 9. Graphs showing the abundance of critical foraminifera taxa in samples C1-6 to C8-3, separated into specimens without (blue bar) and with (red bar) iron staining. 

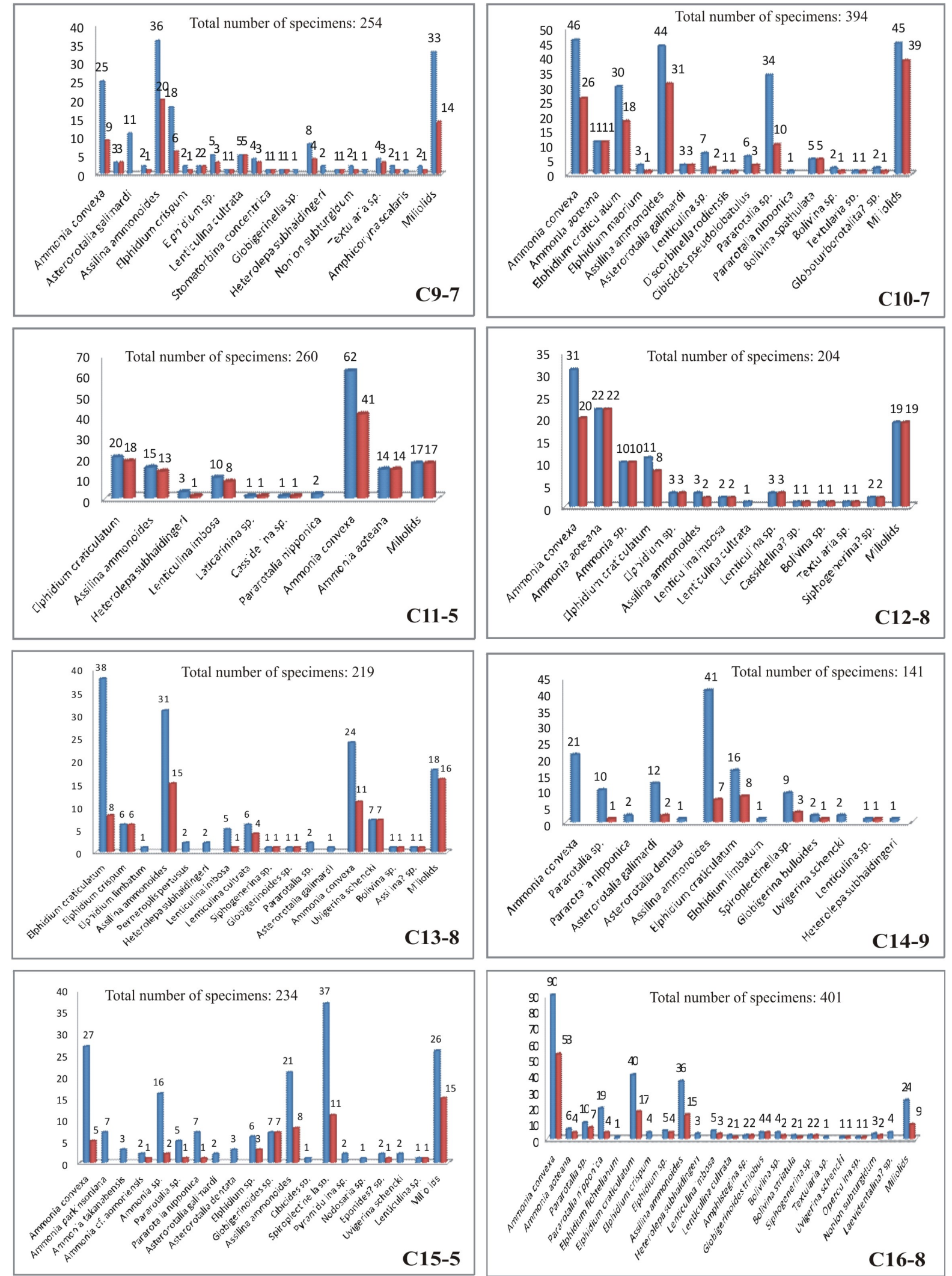

Figure 10. Graphs showing the abundance of critical foraminifera taxa in samples C9-7 to C16-8, separated into specimens without (blue bar) and with (red bar) iron staining. 


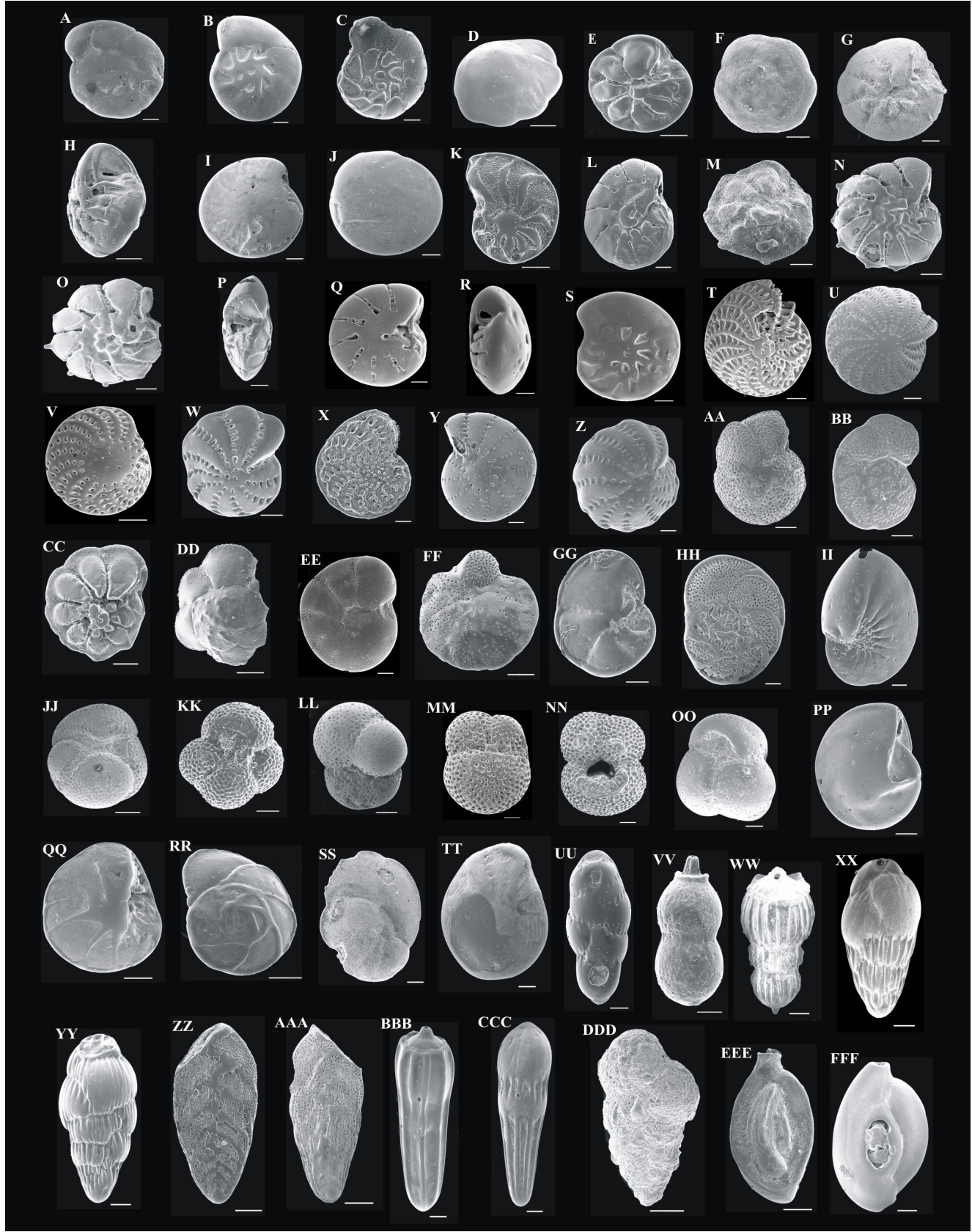

Figure 11. Atlas of foraminifera identified in the study area; all scale bars $100 \mu \mathrm{m}$. A, Ammonia sp., spiral view, sample no. C5-10, Kochak Estuary. B, Ammonia sp., spiral view, sample no. C2-2, Lipar Estuary, C, Ammonia sp., spiral view, C4-4, Gareendar Estuary. D, Ammonia parkinsonia (d'Orbigny, 1839), spiral view, sample no. C15-5, Crab Island. E, Ammonia parkinsonia (d'Orbigny, 1839), umbilical view, sample no. C15-5, Crab Island. F, Ammonia takanabensis (Ishizaki, 1948), spiral view, sample no. C15-5, Crab Island. G, Ammonia takanabensis (Ishizaki, 1948), umbilical view, sample no. C15-5, Crab Island. H, Ammonia convexa (Collins, 1958), apertural view, sample no. C3-9, Lipar Estuary. I, Amphistegina lessoni (d'Orbigny, 1826), umbilical view, sample no. C1-6, Ramin Estuary. J, Amphistegina lessoni (d' Orbigny, 1826), spiral view, sample no. C1-6, Ramin Estuary. K, Assilina ammonoides (Schröter 1783), umbilical view, sample no. C4-4, Gareendar Estuary. L, Challengerella sp., umbilical view, sample no. C4-4, Gareendar Estuary. M, Asterorotalia dentata (Parker and Jones, 1865), umbilical view, sample no. C15-5, Crab Island. N, Asterorotalia gaimardi (d’Orbigny, 1826), umbilical view, sample no. C9-7, Pasabandar. O, Asterorotalia gaimardi (d Orbigny, 1826), umbilical view, sample no. C7-4, Gawater Estuary. P, Asterorotalia gaimardi (d’ Orbigny, 1826), apertural view, sample no. C7-4, Gawater Estuary. Q, Monspeliensina sp., umbilical view, sample no. C1-6, Ramin Estuary. R, Monspeliensina sp., apertural view, sample no. C1-6, Ramin Estuary. S, Monspeliensina sp., side view, sample no. C1-6, Ramin Estuary. T, Elphidium macellum (Fichtel and Moll, 1798), side view, sample no. C1-6, Ramin Estuary. U, Elphidium crispum (Linne, 1758), side view, sample no. C5-10, Kochak Estuary. V, Elphidium crispum (Linne, 1758), side view, sample no. C1-6, Ramin Estuary. W, Elphidium maorium Hayward, 1997, side view, 
sample no. C10-7, Beris Estuary. X, Elphidium fichtelianum (d’ Orbigny, 1846), side view, sample no. C6-8, Gawater Estuary. Y, Elphidium albanii Hayward, 1997, side view, sample no. C6-8, Gawater Estuary. Z, Elphidium excavatum (Terquem, 1875), side view, sample no. C7-4, Gawater Estuary. AA, Lobatula lobatula (Walker and Jacob, 1798), umbilical view, sample no. C9-7, Pasabandar. BB, Lobatula lobatula (Walker and Jacob, 1798), spiral view, sample no. C9-7, Pasabandar.CC, Pararotalia nipponica (Asano, 1936), umbilical view, sample no. C5-10, Kochak Estuary. DD, Pararotalia nipponica (Asano, 1936), spiral view, sample no. C7-4, Gawater Estuary. EE, Anomalinulla glabrata Cushman, 1924, umbilical view, sample no. C1-6, Ramin Estuary. FF, Cibicides sp. spiral view, sample no. C4-4, Gareendar Estuary. GG, Rosalina bradyi (Cushman, 1915), umbilical, sample no. C9-7, Pasabandar. HH, Rosalina bradyi (Cushman, 1915), spiral view, sample no. C9-7, Pasabandar. II, Nonion subturgidum (Cushman, 1924), dorsal view, sample no. C9-7, Pasabandar. JJ, Rosalina orientalis (Cushman, 1925), spiral view, sample no. C2-2, Lipar Estuary. KK, Globigerina bulloides d' Orbigny 1826, spiral view, sample no. C14-9, SE Water Desalination. LL, Globigerinita sp., spiral view, sample no. C2-2, Lipar Estuary. MM, Globigerinoides sp., apertural view, sample no. C1-6, Ramin Estuary. NN, Globigerinoides sp., spiral view, sample no. C7-4, Gawater Estuary. OO, Globigerinoides trilobus (d' Orbigny, 1839), spiral view sample no. C16-8, Park Estuary. PP, Lenticulina australis (Parr, 1950), side view, sample no. C9-7, Pasabandar. QQ, Eponides repandus (Fichtel and Moll, 1798), umbilical view, sample no. C6-8, Gawater Estuary. RR, Eponides repandus (Fichtel and Moll, 1798), spiral view, sample no. C9-7, Pasabandar. SS, Cancris auriculus De Montfort 1808, umbilical view, sample no. C6-8, Gawater Estuary. TT, Peneroplis pertusus (Forskal, 1775), side view, sample no. C2-2, Lipar Estuary. UU, Virgulinella sp., side view, sample no. C3-9, Lipar Estuary. VV, Nodosaria sp., side view, sample no. C4-4, Gareendar Estuary.WW, Pyramidulina? sp., side view, sample no. C15-5, Crab Island. XX, Uvigerina schencki Asano, 1950, side view, sample no. C1-6, Ramin Estuary. YY, Uvigerina schencki Asano, 1950, side view, sample no. C13-8, NW Beris. ZZ, Bolivina spathulata (Williamson, 1858), side view, sample no. C3-9, Lipar Estuary. AAA, Bolivina striatula Cushman 1922, side view, sample no. C15-5, Crab Island. BBB, Siphogenerina sp., side view, sample no. C9-7, Pasabandar. CCC, Siphogenerina sp., side view, sample no. C2-2, Lipar Estuary. DDD, Textularia sp., side view, sample no. C7-4, Gawater Estuary. EEE, Quinqueloculina polygona d'Orbigny, 1839, side view, sample no. C7-4, Gawater Estuary. FFF, Quinqueloculina sp., side view, sample no. C5-10, Kochak Estuary.
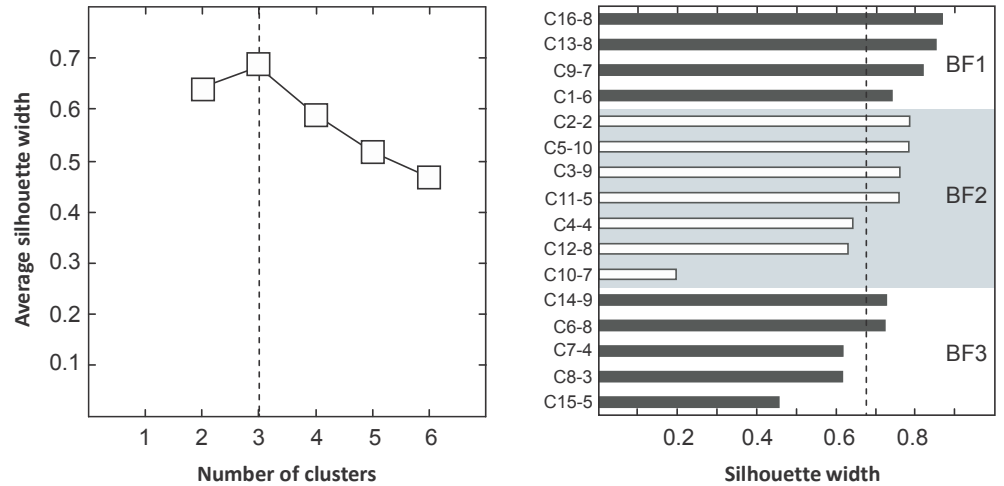

Figure 12. Results of PAM cluster analysis showing three biofacies. a) Average silhouette width for 2 - 6 cluster scenarios. The highest average silhouette width (representing the strongest structure) for each cluster is indicated by a hashed line. b) Silhouette plots of stained and non-stained foraminifera divided into three clusters (highest average silhouette width). Grey and white areas differentiate between clusters and the average silhouette width is indicated by a dashed line.

\section{Discussion}

Okal et al. (2015) report the result of a 2010 survey of the effects on the Iranian coastline of the tsunami which followed the earthquake of 28 November $1945\left(\mathrm{M}_{\mathrm{O}}=2.8 \times 10^{28}\right.$ dyn $\left.\mathrm{cm} ; \mathrm{M}_{\mathrm{W}}=8.2\right)$ which accompanied with the tsunami waves, resulted from an ancillary phenomenon, such as a landslide operated by the earthquake. Also, Shah-Hosseini et al. (2011) reported 58 coastal boulders attesting to large waves along the rocky coast of Iranian Makran from Chahbahar to Lipar (Fig. 1) which are mostly rectangular and composed of biogenic calcarenite deriving from the present coastal platform. They have morphological features include supratidal karstic pools, sharp broken edges and fractures, and some of them contain boreholes and shells of marine bivalves, suggesting detachment and transportation from the subtidal zone (ShahHosseini et al., 2011). The boulders, weighing up to $18 \mathrm{t}$, were found up to $6 \mathrm{~m}$ above present mean sea level and up to $40 \mathrm{~m}$ from the present shoreline. Shah-Hosseini et al. (2011) believe tsunami wave height of $4 \mathrm{~m}$ is enough to detach all the boulders from the rocky coast and transport them inland, and a tsunamigenic origin for boulder deposits is most possible which probably generated by large earthquakes at the Makran subduction zone.

In addition to the issues discussed above, the uppermost coastal sediments of the Oman Sea (Chabahar Bay to the middle part of Gawater Bay) contain a significant percentage of foraminifera which are mostly corroded and show iron staining, and could be used as an overwash indicator in 
coastal and lagoonal environments. Thses sediments indicate an event layer which can be either stormor tsunami generated. The presence of heavy boulders in the coast of Iranian Makran which exclusively found in the tsunami deposit could be confirmed a tsunami event.

The abundance and significant foraminiferal species in the studied samples are Ammonia convexa, A. aoteana, A. tepida, A. inflate, Amphistegina lessonii, Assilina ammonoides Asterorotalia galimardi, Elphidium craticulatum, E. crispum, Pararotalia nipponica, Spiroplectinella sp., Lenticulina sp., planktic species (e.g. Globigerinoides, Globigerinita, Globorotalia) and various miliolids. The most useful taxa for recognizing a marine incursion (e.g. tsunami or storm) are abundant Amphistegina spp., Ammonia inflata, Elphidium advenum and planktic species, with these groups expected to be highly concentrated through transport and sorting within an event bed.

Foraminiferal taphonomy has previously been used as an overwash indicator in coastal environments (Pilarczyk et al., 2014; Rubin et al., 2017; Kosciuch et al., 2018). The abundance of miliolids, Ammonia tepida, Ammonia parkinsoniana, and Elphidium gerthi suggests these taxa are the dominant foraminifera living in the coastal bay, an interpretation consistent with other similar coastal environments (e.g. Murray, 1991; Debenay et al., 2001; Lezine et al., 2002; Ghosh et al., 2009).

Most of the taxa listed in this study are considered indicative of coastal bay and lagoonal environments. This idea is consistent with similar coastal environments where these taxa also dominate assemblages (e.g. Murray, 1991; Debenay et al., 2001, Debenay, 2012; Lezine et al., 2002; Ghosh et al., 2009).

In this study, the abundances foraminifera such as Amphistegina, Ammonia, Elphidium, and planktics which are predominantly found in the shallow marine area are useful taxa for recognizing a marine overwash (e.g. tsunami or storm). The existence of iron staining on foraminiferal tests and the observed percentages of corrosion may be related to upwelling with a marine incursion such as tsunami or storm, and the effects of erosion and corrosion under oxidizing conditions. The foraminiferal analysis was able to distinguish the tsunami unit based on shell taphonomy and particle size analysis. Therefore, foraminifera appear to be the most useful parameters for assessing overwash events along the Iranian coast.

\section{Conclusions}

Sediments studied from the Iranian coast of the Oman Sea appear to have been transported from the shallow marine area into the modern coastal platforms, sandy beaches, and mudflats by a marine incursion such as a tsunami or storm. This transport of sediments is supported by the foraminiferal results. In this study, the surface distribution of foraminifera was examined in various subenvironments of the Makran coast between Chabahar and Gawater Bays, Oman Sea. These foraminifera obtained from these locations are dominated by subtidal, intertidal, and supratidal species, with minor abundances of planktic taxa, and showing iron staining and corrosion. By PAM cluster analysis were determined three foraminiferal assemblages existing within the Makran coastal zone: subtidal, intertidal, and supratidal. Samples collected from intertidal locations $\left(\mathrm{BF}_{1}\right)$ are characterized by high abundances of heavily corroded individuals, whereas samples collected from supratidal locations $\left(\mathrm{BF}_{2}\right)$ are dominated by high abundances of iron-stained and heavily corroded individuals, and samples collected from subtidal locations $\left(\mathrm{BF}_{3}\right)$ are characterized by low abundances of iron-stained individuals and high abundances of moderately corroded individuals. This suggests that analyzing modern distributions of fossil foraminifera in surface samples will enable an improved interpretation of the 1945 deposit.

\section{Acknowledgments}

The present research has been carried out at the School of Geography and Earth Sciences, McMaster University, Canada which is gratefully acknowledged. The authors would like to deeply thank the Iranian National Institute for Oceanography and Atmospheric Science (INIOAS)-Gulf of Oman and Indian Ocean Research Center (Chabahar) for logistic support in the field, M.A. Hamzeh (Graduate University of Advanced Technology \& INIOAS), $\mathrm{H}$. and $\mathrm{M}$. Afarin (INIOAS) for their assistance in the field, A. Naderi-Beni (INIOAS), M. Shah-Hosseini (AixMarseille University), and D. Jahani (Islamic Azad University, North Tehran Branch) for their useful comments and suggestions, J. Murray (University of Southampton) and J.P. Debenay (University of Angers) for reviewing and valuable suggestions on 
foraminiferal samples, and S.K. Martin (Geological Survey of Western Australia), A. Matsuoka (Niigata University) and two anonymous reviewers for providing constructive comments on the manuscript. The photomicrographs of foraminifers were obtained by scanning electron microscope (SEM) with the kind help of G. de Silveira at the Canadian for Electron Microscopy, McMaster University, which we gratefully acknowledge.

\section{References}

Akimoto, K., Matsui, C., Shimokawa, A., Furukawa, K., 2002. Atlas of Holocene benthic foraminifers of Shimabara Bay, Kyushu, Southwest Japan. The Kagoshima University Museum Monographs Publication, 2: 1-112.

Ambraseys, N.N., Melville, C.P., 1982. A History of Persian Earthquakes. Cambridge: Cambridge University Press, Britain, 1-213.

Bhalla, S.N., Khare, N., Shanmukha, D.H., Henriques, P.J. 2007. Foraminiferal studies in nearshore regions of the western coast of India and Laccadives Islands: a review: Indian Journal of Marine Sciences, 36: 272-287.

Byrne, D.E., Sykes, L.R., Davis, D.M., 1992. Great thrust earthquakes and seismic slip along the plate boundary of the Makran Subduction Zone. Journal of Geophysical Research, 97: 449-478.

Cherif, O.H., Al-Ghadban, A., Al-Rifaiy, I.A., 1997. Distribution of foraminifera in the Arabian Gulf. Micropaleontology, 43: 253-280.

Cimerman, F., Langer, M.R., 1991. Mediterranean Foraminifera. Slovenska Akademija Znanosti in Umetnosti, 1-118.

Debenay, J.P., 2012. A guide to 1000 foraminifera from Southwestern Pacific New Caledonia. IRD/MNHN Scientific Museum publications, Paris, 1-378.

Debenay, J.-P., Geslin, E., Eichler, B.B., Duleba, W., Sylvestre, F., Eichler, P., 2001. Foraminiferal assemblages in a hypersaline lagoon, Araruama (R.J.) Brazil. Journal of Foraminiferal Research, 31: 133-151.

Dominey-Howes, D.T.M., Humphreys, G.S., Hesse, P.P. 2006. Tsunami and palaeotsunami depositional signatures and their potential value in understanding the late-Holocene tsunami record. The Holocene, 16 (8): 1095-1107.

Donato, S.V., Reinhardt, E.G., Boyee, J.I., Pilarczyk, J.E., Jupp, B.P., 2009. Particle-size distribution of inferred tsunami deposits in Sur Lagoon, Sultanate of Oman. Marine Geology, 257: 54-64.

Donato, S.V., Reinhardt, E.G., Boyee, J.I., Rothaus, R., Vosmer, T., 2008. Identifying tsunami deposits using bivalve shell taphonomy. Geology, 36: 199-202.

El-Nakhal, H., 1990. Geographical distribution of the foraminiferal genus Agglutinella. Micropaleontology, 36: 86-87.

Ghosh, A., Saha, S., Saraswati, P.K., Banerjee, S., Burley, S., 2009. Intertidal foraminifera in the macro-tidal estuaries of the Gulf of Cambay: implications for interpreting sea-level change in paleo-estuaries. Marine and Petroleum Geology, 26: $1592-1599$.

Goff, J., Lamarche, G., Pelletier, B., Chague-Goff, C., Strotz, L., 2011. Predecessors to the 2009 South Pacific tsunami in the Wallis and Futuna archipelago. Earth-Science Reviews, 107: 91-106.

Hamzeh, M.A., Jokar, R. Beskaleh, Gh.R., 2014. Classification of Iranian Oman Sea coasts on sedimentology and geomorphology (Chabahar Bay to Gawater Bay). 32 ${ }^{\text {nd }}$ National and the $1^{\text {st }}$ International Geosciences Congress Abstract, Tehran, p. 143 (in Persian).

Hamzeh, M.A., Okal, E.A, Ghasemzadeh, J., Beskaleh, Gh.R., 2013. Assessing effects of the tsunami 1945 Pakistan on the Iranian Makran Coast. First National Conference on the development of Makran coast and sea power Iran, Chabahar, paper, 1123: 1-9 (in Persian).

Hawkes, A.D., Horton, B.P., 2012. Sedimentary record of storm deposits from Hurricane Ike, Galveston and San Luis Islands, Texas. Geomorphology, 171-172: 180-189.

Heidarzadeh, M., Pirooz, M.D., Zaker, N.H., Synolakis, C.E., 2008b. Evaluating tsunami hazard in the northwestern Indian Ocean. Pure and Applied Geophysics, 165: 2045-2058.

Heidarzadeh, M., Pirooz, M.D., Zaker, N.H., Yalciner, A.C., 2009. Preliminary estimation of the tsunami hazards associated with Makran subduction zone at the north-western Indian Ocean. Natural Hazards, 48: 229-243.

Heidarzadeh, M., Pirooz, M.D., Zaker, N.H., Yalciner, A.C., Mokhtari, M., Esmaeili, A., 2008a. Historical tsunami in the Makran Subduction Zone of the southern coasts of Iran and Pakistan and results of numerical modeling. Ocean Engineering, 35: 774-786.

Hippensteel, S.P., Martin, R.E., Nikitina, D., Pizzuto, J.E., 2000. The formation of Holocene marsh foraminiferal assemblages, middle Atlantic Coast, U.S.A.: implications for Holocene sea-level change. Journal of Foraminiferal Research, 30: 272-293.

Hottinger, L., Halicz, E., Reiss, Z., 1993. Recent foraminiferida from the Gulf of Aqaba, Red Sea. Slovenska Akademija Znanosti in Umetnosti, 1-179.

Jaiswal, R.K., Singh, A.P., Rastogi, B.K., 2009. Simulation of the Arabian Sea Tsunami propagation generated due to 1945 Makran Earthquake and its effect on western parts of Gujarat (India). Natural Hazards, 48: 245-258. 
Kaufman, L., Rousseeuw, P.J., 1990. Finding Groups in Data: An Introduction to Cluster Analysis. A John Wiley and Sons, INC. Publication, 1-342.

Kosciuch, T.J., Pilarczyk, J.E., Hong, I., Fritz, H.M., Horton, B.P., Rarai, A., Harrison, M.J., Jockley, F.R., 2018. Foraminifera reveal a shallow near shore origin for overwash sediments deposited by Tropical Cyclone Pam in Vanuatu (South Pacific). Marine Geology, 396: 171-185.

Lézine, A., Saliége, J. Mathieu, R., Tagliatela, T., Mery, S., Charpentier, V., Cleuziou, S., 2002. Mangroves of Oman during the late Holocene: climatic implications and impact on human settlements. Veg. Hist. Archaeobot, 11: $221-232$.

Loeblich, A.R., Tappan, H., 1988. Foraminiferal Genera and their Classification. Van Nostrand Rienhold Company, New York, 1-970.

Moghaddasi, B., Nabavi, S.M.B., Vosoughi, G., Fatemi, S.M.R., Jamili, S., 2008. Abundance and distribution of benthic foraminifera in the Northern Oman Sea (Iranian Side) continental shelf sediments. Research Journal of Environmental Sciences, 3: 210-217.

Mokhtari, M., Abdullahi Fard, I., Hessami, K., 2008. Structural elements of the Makran region, Oman Sea and their potential relevance to tsunami genesis. Natural Hazard, 47: 185-199.

Murray, J.W., 1965. The foraminiferida of the Persian Gulf. 2. The Abu Dhabi region. Palaeogeography, Palaeoclimatology, Palaeoecology, 1: 307-332.

Murray, J.W., 1966a. The foraminiferida of the Persian Gulf. 3. The Halat Al Bahrani region. Palaeogeography, Palaeoclimatology, Palaeoecology, 2: 59-68.

Murray, J.W., 1966b. The foraminiferida of the Persian Gulf. 4. Khor Al Bazam. Palaeogeography, Palaeoclimatology, Palaeoecology 2: 153-169.

Murray, J.W., 1966c. The foraminiferida of the Persian Gulf. 5. The shelf of the Trucial Coast. Palaeogeography, Palaeoclimatology, Palaeoecology, 2: 267-278.

Murray, J.W., 1991. Ecology and Paleoecology of Benthic Foraminifera. John Wiely and Sons Inc. 1-397.

Nigam, R., Khare, N., 1995. Recent foraminifera along west coast of India: retrospect and prospect. Journal of the Indian Academy of Geoseciene, 38: 7-24.

Okal, E.A., Fritz, H.M., Hamzeh, M.A., Ghasemzadeh, J., 2015. Field survey of the 1945 Makran and 2004 Indian Ocean Tsunamis in Baluchistan, Iran. Pure and Applied Geophysics 172: 3343-3356.

Okal, E.A., Synolakis, C.E., 2008. Far-field tsunami hazard from mega-thrust earthquakes in the Indian Ocean. Geophysical Journal International, 172: 995-1015.

Pararas-Carayannis, G., 2006. The potential for tsunami generation along the Makran Subduction Zone in earthquake and tsunami of November 28, 1945. Science of Tsunami Hazard, 24 (5): 358-384.

Payande, A.R., Niksokhan, M.H., Naserian, H., 2015. Tsunami hazard assessment of Chabahar Bay related to megathrust seismogenic potential of the Makran subduction zone. Natural Hazard, 76: 161-176.

Pham, D.T., Gouramanis, C., Switzer, A.D., Rubin, C.M., Jones, B.G., Jankaew, K. and Carr, P.F., 2018. Elemental and mineralogical analysis of marine and coastal sediments from Phra Thong Island, Thailand: Insights into the provenance of coastal hazard Deposits. Marine Geology, 396: 79-99.

Pilarczyk, J.E., Dura, T., Horton, B.P., Engelhart, S.E., Kemp, A.C., Sawai, Y., 2014. Microfossils in coastal environments as indicators of paleo- earthquakes, tsunamis and storms. Paleogeography, Palaeoclimatology, Palaeoecology, 413: 144-157.

Pilarczyk, J.E., Horton, B.P., Soria J.A., Switzer, A.D., Siringan, F., Fritz, H.M., Khan, N.S., Ildefonso, S., Doctor, A.A., Garcia, M.L., 2016. Micropaleontology of the 2013 Typhoon Haiyan overwash sediments from the Leyte Gulf, Philippines, Sedimentary Geology, 339: 104-114.

Pilarczyk, J.E., Horton, B.P., Writer, R.C., Vane, C.H., Goff, J., Chaqué-Goff, C., 2012. Sedimentary and foraminiferal evidence of the 2011 Tohoku-oki tsunami on the Sendai coastal plain, Japan. Sedimentary Geology, 281: 78-89.

Pilarczyk, J.E., Reinhardt, E.G., 2012. Testing foraminiferal taphonomy as a tsunami indicator in a shallow arid system lagoon: Sur, Sultanate of Oman. Marine Micropaleontology, 295-298: 128-136.

Pilarczyk, J.E., Reinhardt, E.G., Boyce, J.I., Schwarcz, H.P., Donato, S.V., 2011. Assessing surficial foraminiferal distributions as an overwash indicator in Sur Lagoon, Sultanate of Oman. Marine Micropaleontology, 80: 62-73.

Reddy, K.R., Rao, R.J., 1984. Foraminifera-salinity relationship in the Pennar Estuary, India. Journal of Foraminiferal Research, 14: 115-119.

Regard, V., Bellier, O., Thomas, J.C., Bourlès, D., Bonnet, S., Abbassi, M.R., Braucher, R., Mercier, J., Shabanian, E., Soleymani, Sh., Feghhi, Kh., 2005. Cumulative right-lateral fault slip rate across the Zagros-Makran transfer zone: role of the Minab-Zendan fault system in accommodating Arabia-Eurasia convergence in southeast Iran. Geophysical Journal International, 162: 177-203.

Reyss, J.L., Pirazzoli, P.A., Haghipour, A., 1998. Quaternary marine terraces and tectonic uplift rates on the south coast of Iran. In: Stewart, I.S., Vita-Finzi, C. (Eds.), Coastal Tectonics: Geological Society of 
London, Special Publication, 146: 225-237.

Rubin, C.M., Horton, B.P., Pilarczyk, J.E., Daly, P., Parnell, A.C. 2017. Highly variable recurrence of tsunamis in the 7,400 years before the 2004 Indian Ocean tsunami. Nature Communications 8 (16019), doi: 10.1038/ncomms16019.

Scott, D.B., Collins, E.S. Gayes, P.T., Wright, E., 2003. Records of prehistoric hurricanes on the South Carolina coast on micropaleontological and sedimentological evidence, with a comparison to other Atlantic Coast records. Geological Society of America Bulletin, 115: 1027-1039.

Shah-Hosseini, M., Morhange, C., Baderi Beni, A., Marriner, N., Lahijiani, H., Hamzeh, M.A., Sabatier, F., 2011, Coastal boulders as evidence for high-energy waves on the Iranian coast of Makran, Marine Geology, 290: 17-28.

Sieh, K., Daly, P., Mckinnon, E.E., Pilarczyk, J.E., Chiang, H.W., Horton, B., Rubin, C.M., Shen, C.C., Ismail, N., Vane, C.H., Feener, R.M., 2015. Penultimate predecessors of the 2004 Indian Ocean tsunami in Aceh, Sumatra: Stratigraphic, archeological, and historical evidence, Journal of Geophysical Research. Solid Earth, 120: 308-325, doi:10.1002/2014JB011538.

Sugawara, D., Minoura, K., Nemoto, N., Tsukawaki, S., Goto, K., Imamura, F., 2009. Foraminiferal evidence of submarine sediment transport and deposition by backwash during the 2004 Indian Ocean tsunami. Island Arc, 18: $513-$ 525. 